\title{
A SYSTEMATIC STUDY OF THE FINAL MASSES OF GAS GIANT PLANETS
}

\author{
Takayuki Tanigawa and Masahiro Ikoma \\ Department of Earth and Planetary Sciences, Tokyo Institute of Technology, Ookayama, \\ Meguro-ku, Tokyo 152-8551, Japan \\ tanigawa@geo.titech.ac.jp
}

\begin{abstract}
We construct an analytic model for the rate of gas accretion onto a planet embedded in a protoplanetary disk as a function of planetary mass, disk viscosity, disk scale height, and unperturbed surface density in order to study the longterm accretion and final masses of gas giant planets. We first derive an analytical formula for surface density profile near the planetary orbit from considerations of the balance of force and the dynamical stability. Using it in the empirical formula linking surface density with gas accretion rate that is derived based on hydrodynamic simulations of Tanigawa \& Watanabe (2002, ApJ 586, 506), we then simulate the mass evolution of gas giant planets in viscously-evolving disks. We finally determine the final mass as a function of semi-major axis of the planet. We find that the disk can be divided into three regions characterized by different processes by which the final mass is determined. In the inner region, the planet grows quickly and forms a deep gap to suppress the growth by itself before disk dissipation. The final mass of the planet in this region is found to increase with the semi-major axis in a similar way to the mass given by the viscous condition for gap opening, but the former is larger by a factor of approximately 10 than the latter. In the intermediate region, viscous diffusion of the disk gas limits the gas accretion before the planet form a deep gap. The final mass can be up to the disk mass, when disk viscous evolution occurs faster than disk evaporation. In the outer region, planets capture only tiny amounts of gas within the lifetime of the disk to form Neptune-like planets. We derive analytic formulae for the final masses in the different regions and the locations of the boundaries, which are helpful to gain a systematic understanding of the masses of gas giant planets.
\end{abstract}

Subject headings: methods: analytical — methods: numerical — solar system: formation 


\section{Introduction}

A fundamental but unresolved issue with planet formation is how the mass of a giant planet is fixed. In the solar system there are four giant planets, which are characterized by their massive hydrogen/helium envelopes. Jupiter and Saturn consist mostly of hydrogen/helium, while Uranus and Neptune are mostly of ice but have significant amounts of hydrogen/helium. The giant planets are different in mass: Jupiter's mass is $1 \times 10^{-3} M_{\odot}\left(=M_{J}\right)$, Saturn's mass is $\sim 0.3 M_{J}$, and Uranus' and Neptune's masses are $\sim 0.05 M_{J}$. Furthermore, the extrasolar planets detected so far also range in mass from about one Neptune mass to $\sim 10 M \sqrt{1}$; those planets are believed to have massive hydrogen/helium envelopes like the giant planets in the solar system.

There are two competing ideas for the formation of giant planets, the core accretion model (e.g., Mizuno 1980; Bodenheimer \& Pollack 1986) and the disk instability model (e.g., Cameron 1978; Boss 1989). In the core accretion model, a rocky/icy core first forms through collisional aggregation of planetesimals, followed by envelope formation due to substantial accretion of gas from the circumstellar (protoplanetary) disk. In the disk instability model, density fluctuation of the disk gas grows to form a gaseous planet, followed by core formation due to sedimentation of heavy elements in its interior. The advantages and disadvantages of both models are discussed in several literatures (e.g., Boss 2002), which is not repeated here. In this paper we consider giant planet formation in the context of the core accretion model.

In the core accretion model, the process of the accumulation of the envelopes is divided into two phases, the subcritical-accretion and supercritical-accretion phases, in terms of the dominant energy source. The transition from the former to the latter occurs when the mass of a core reaches a critical value. In the subcritical-accretion phase, incoming planetesimals supply energy to the envelope so that the envelope is in the hydrostatic equilibrium. The core accretion thus controls the gas accretion in this phase. The phase 2 found by Pollack et al. (1996) is a part of the subcritical-accretion phase. In the supercritical-accretion phase, the energy supplied by planetesimals is insufficient to keep the hydrostatic structure of the envelope, so that the envelope substantially contracts and releases its gravitational energy, resulting in runaway accretion of the disk gas.

The supercritical-accretion phase can be further divided into two subphases. In the former subphase, the gas accretion is controlled by contraction of the envelope and thus occurs on the Kelvin-Helmholtz timescale (Bodenheimer \& Pollack 1986; Pollack et al. 1996;

\footnotetext{
${ }^{1}$ http://exoplanet.eu/
} 
Ikoma, Nakazawa, \& Emori 2000; Ikoma \& Genda 2006). However, because the gas accretion driven by the envelope contraction accelerates rapidly with time, the supply of the disk gas inevitably becomes unable to keep up with the demand of the contracting envelope. Thus, in the latter subphase, the disk-gas supply limits the gas accretion (Tanigawa \& Watanabe 2002, hereafter TW02).

In this paper we focus on the gas accretion in the latter subphase of the supercriticalaccretion phase, i.e., the phase in which the Kelvin-Helmholtz contraction of the envelope is under way but the gas accretion is limited by disk-gas supply. After the onset of the runaway gas accretion (i.e., in the supercritical-accretion phase), the planet always experiences the limited gas supply (i.e., the latter subphase) by the end of its growth. Although there is an idea that the masses of Uranus and Neptune were fixed in the subcritical-accretion phase because of dissipation of the disk gas (Pollack et al. 1996), we will explore another possibility that the masses of those planets were fixed in the supercritical-accretion phase.

One idea is that the mass of a giant planet is completely fixed when the planet opens a gap in the disk. From a physical point of view, the gap opens when the gravitational scattering by the planet overwhelms both viscous diffusion (the viscous condition) and push due to pressure gradient (the thermal condition) (e.g., Lin \& Papaloizou 1993). In normal protoplanetary disks, the final mass of a giant planet is determined by the thermal condition (e.g., Ida \& Lin 2004), which is equivalent to the condition that the Hill radius is equal to the disk scale height at the planet's location. The final mass is thus determined only by temperature of the disk gas for given stellar mass and planet's location. In protoplanetary disks similar to the minimum-mass solar nebula (Hayashi 1981), the thermal condition yields the final mass of approximately one Jupiter mass at $5 \mathrm{AU}$, but predicts that the masses of giant planets increase with the semi-major axis, which is in clear contradiction to the current configuration of the solar system. Furthermore, because, in reality, the gap is not vacuum but a low-density region, continuous gas accretion through the gap takes place, as suggested by several studies (e.g., Artymowicz \& Lubow 1996). The effects of the subsequent accretion on the final masses of giant planets have been poorly understood.

Another idea is that gas accretion is truncated by depletion of the disk gas before gap opening (e.g., viscous dissipation and photo-evaporation of the disk gas). This means that the final mass of a giant planet is the total mass of the disk gas that the planet has captured until the disk gas dissipates completely. This may be responsible for the current configuration of the solar system, since it takes longer time for outer giant planets to accrete principally because of their long orbital periods. However, there have been no quantitative studies of this possibility.

The purpose of this paper is to gain a systematic understanding of how the final mass of 
a giant planet is determined. Specifically, we clarify (1) how much the mass of an accreting giant planet increases via slow, continuous accretion through the gap, (2) when and where disk dissipation dominates gap opening, and finally (3) how the final masses of giant planets depend on several disk parameters. To do so, we simulate the long-term accretion of a giant planet in a self-consistent fashion. The essential ingredients of the modeling are global viscous evolution of the disk and the flow pattern of the accreting gas, as described below; especially, the latter effect was completely neglected by previous studies. Furthermore, we derive analytical formulas for the final masses of giant planets and the boundaries between several different regimes.

During the viscous evolution of the disk, gas radially migrates from outer regions to the site of giant planet formation. The mass flux limits gas accretion onto giant planets in some situations. Because of limited CPU speed of computers, azimuthally-averaged one-dimensional simulations of the disk evolution are inevitable to follow the long-term accretion. Several previous studies simulated the radial mass flux in viscously evolving disks and then calculated gas accretion rate onto giant planets. Lecar \& Sasselov (2003) and Guillot \& Hueso (2006) also simply assumed that gas accretion rate onto giant planets is always proportional to the viscous mass flux. Veras \& Armitage (2004) and Alibert, Mordasini, \& Benz (2004) used an approximate formula for gas accretion rate that fits the results of twodimensional hydrodynamic simulations of disk gas with an embedded planet done by Lubow, Seibert, \& Art (1999) and D'Angelo, Henning, \& Kley (2002). However, the formula was derived based on a limited number of numerical simulations. Hence, it is in question whether the formula applies in a variety of situations.

To understand the detailed pattern of accretion flow and to obtain the gas accretion rate onto the planet, several workers (e.g., Klev 1999; Lubow. Seibert. \& Artvmowicz 1999; D'Angelo, Henning, \& Kley 2002; Bate et al. 2003; D’Angelo, Kley, \& Henning 2003) performed two- or three-dimensional hydrodynamic simulations of interaction between a planet and disk gas. Since they simulated global disks, the effect of gap formation on accretion rate was automatically included. However, because of the time-consuming simulations, they did not simulate the growth of giant planets on timescales of the viscous disk evolution.

This study is clearly different from the previous studies in that this study includes the effects of the flow pattern of the accreting gas in simulating long-term growth of planets embedded in globally evolving disks. To do so, we use the empirical relation between gas accretion rate and unperturbed surface density that was derived by TW02 (see $\S 3$ for the details). They carried out detailed two-dimensional hydrodynamic simulations of gas accretion flow onto a planet. They demonstrated the importance of the local flow pattern on the accretion rate. 
In this paper, we first derive an analytical formula for radial surface density distribution of protoplanetary disks with an embedded planet in $\S 2$. Next, we describe how to include the effect of the flow pattern of accreting gas, following TW02, to calculate gas accretion rate onto giant planets in $\S 3$. In $\S 4$, we simulate the long-term accretion of giant planets, based on the prescription given in $\S \S 2$ and 3 , and obtain the final masses of giant planets for wide ranges of several disk parameters. Also, we derive analytical approximate formulas for the final masses of giant planets and the boundaries between several regimes. Finally, we conclude this paper in $\S 5$.

\section{Surface density}

We consider a planet embedded in a gas disk, both of which are assumed to rotate with the Keplerian velocity around a star. We derive an approximate formula for azimuthallyaveraged surface density as a function of radial distance to the central star, $r$. We adopt a local coordinate system in which all quantities except the surface density are independent of $r$. We assume steady states and no mass flow toward the central star. We do not consider migration of the planet. The validity of these assumptions are discussed in $\$ 4.4$.

We employ a local coordinate system co-rotating with the planet on a circular Keplerian orbit around the central star (a local shearing-sheet approximation). The origin of the coordinate system is the planet's position. The $x$ axis is on the line from the star through the planet; the direction of the $y$ axis is the same as that of the velocity vector of the planet. The equation of motion of the disk gas in the $y$ direction in this coordinate system is given by

$$
\frac{\partial}{\partial t}\left(\Sigma v_{y}\right)+\frac{\partial}{\partial x}\left(\Sigma v_{y} v_{x}\right)=\frac{\partial}{\partial x}\left(\nu \Sigma \frac{\partial v_{y}}{\partial x}\right)+\Sigma \dot{v}_{y}
$$

where $\Sigma$ is surface density, $v_{x}$ and $v_{y}$ are velocities in the $x$ and $y$ directions, $\nu$ is viscosity coefficient, and $\dot{v}_{y}$ is force acting on gas per unit mass. Here we have omitted the advection term in the $y$ direction because of axisymmetry. We also eliminate all the terms on the left-hand side of this equation on the assumptions of steady states $(\partial / \partial t=0)$ and no mass flow $\left(v_{x}=0\right)$. Hereafter we consider only $x>0$ because of symmetry.

To derive an analytical formula for $\Sigma(x)$, we assume that $v_{y}$ is equal to the Keplerian velocity, $v_{y}=-(3 / 2) \Omega_{\mathrm{p}} x$ where $\Omega_{\mathrm{p}}$ is the Keplerian angular velocity at the planet's position. This assumption will be found to be reasonable later. Equation (11) is thus transformed into

$$
-\frac{3}{2} \nu \Omega_{\mathrm{p}} \frac{\partial \ln \Sigma}{\partial x}+\dot{v}_{y}=0
$$

The gravity of the planet perturbs the motion of the disk gas. We follow the impulse 
prescription (e.g., Lin \& Papaloizou 1979) that approximates scattering of the disk gas as small-angle scattering of a particle by a point-mass object. On the prescription, $\dot{v}_{y}$ is given by

$$
\dot{v}_{y}=\frac{4}{9 \pi}\left(\frac{M_{\mathrm{p}}}{M_{*}}\right)^{2} r_{\mathrm{p}}^{5} \Omega_{\mathrm{p}}^{2} x^{-4},
$$

where $r_{\mathrm{p}}$ is the distance of the planet from the central star and $M_{\mathrm{p}}$ and $M_{*}$ are the masses of the planet and the central star, respectively. This approximation is inappropriate for $x \lesssim$ $\min \left(2 r_{\mathrm{H}}, r_{\mathrm{p}} \sqrt{\left(M_{\mathrm{p}} / M_{*}\right) / h}\right)$, where $r_{\mathrm{H}} \equiv r_{\mathrm{p}}\left(M_{\mathrm{p}} / 3 M_{*}\right)^{1 / 3}$ namely, the Hill radius, because this region is what is called the horse-shoe region, in which gas is not pushed away from the planet. However, since the gas in the horse-shoe region has no contribution to the accretion flow of interest (see in \$3), we neglect such a region. Note that Crida, Morbidelli, \& Masset (2006) proposed a more elaborate formula for the torque (a $y$-direction force in our formulation) that includes the radial transfer of torque through waves, which they found in their twodimensional hydrodynamic simulations. However, since the formula is unable to apply for wide ranges of the parameters, as they mentioned, we do not use their formula in this study.

Substituting equation (3) into equation (2), we obtain

$$
\Sigma(x)=\Sigma_{\infty} \exp \left[-\left(\frac{x}{\ell}\right)^{-3}\right] \equiv \Sigma_{\text {vis }}(x),
$$

where $\Sigma_{\infty}$ is the surface density at infinity (i.e., far away from the planet's orbital radius) and $\ell$ is defined as

$$
\begin{aligned}
\ell & \equiv\left[\frac{8}{81 \pi}\left(\frac{\nu}{r_{\mathrm{p}}^{2} \Omega_{\mathrm{p}}}\right)^{-1}\left(\frac{M_{\mathrm{p}}}{M_{*}}\right)^{2}\right]^{1 / 3} r_{\mathrm{p}} \\
& =0.146\left(\frac{\nu}{10^{-5} r_{\mathrm{p}}^{2} \Omega_{\mathrm{p}}}\right)^{-1 / 3}\left(\frac{M_{\mathrm{p}}}{10^{-3} M_{*}}\right)^{2 / 3} r_{\mathrm{p}}
\end{aligned}
$$

This surface density profile is basically the same as that derived by Lubow \& D'Angelo (2006), except for the value of the coefficient.

The dynamical stability of the surface density profile given by equation (4) must be checked using the well-known Rayleigh criterion (e.g., Chandrasekhar 1961). For the surface density profile to be stable, in the local coordinate system,

$$
\frac{\partial v_{y}}{\partial x} \geq-2 \Omega_{\mathrm{p}}
$$

must be fulfilled. The velocity $v_{y}$ can be obtained by solving the equation of motion for the $x$ component,

$$
3 \Omega_{\mathrm{p}}^{2} x+2 \Omega_{\mathrm{p}} v_{y}-c^{2} \frac{\partial \ln \Sigma}{\partial x}=0,
$$


where $c$ is sound speed. Substituting equation (44) into equation (8), we obtain

$$
v_{y}=-\frac{3}{2} \Omega_{\mathrm{p}} x\left[1-\left(\frac{h}{\ell}\right)^{2}\left(\frac{x}{\ell}\right)^{-5}\right],
$$

where $h$ is the disk scale height defined as $h \equiv c / \Omega_{\mathrm{p}}$. From equations (7) and (9), we find that condition (7) is not fulfilled for $x<x_{\mathrm{m}}$, where

$$
\begin{aligned}
x_{\mathrm{m}} & \equiv 12^{1 / 5}\left(\frac{h}{\ell}\right)^{2 / 5} \ell=12^{1 / 5}\left(\frac{\ell}{h}\right)^{3 / 5} h \\
& =0.207\left(\frac{h}{0.1 r_{\mathrm{p}}}\right)^{2 / 5}\left(\frac{\nu}{10^{-5} r_{\mathrm{p}}^{2} \Omega_{\mathrm{p}}}\right)^{-1 / 5}\left(\frac{M_{\mathrm{p}}}{10^{-3} M_{*}}\right)^{2 / 5} r_{\mathrm{p}} .
\end{aligned}
$$

In the region $x \leq x_{\mathrm{m}}$, the density gradient thus has to be small enough to satisfy the Rayleigh condition: the density profile would be relaxed to be marginally stable for the Rayleigh condition. Integrating $\partial v_{y} / \partial x=-2 \Omega_{\mathrm{p}}$ inward from $x_{\mathrm{m}}$ we obtain

$$
v_{y}=v_{y, \mathrm{~m}}-2 \Omega_{\mathrm{p}}\left(x-x_{\mathrm{m}}\right)
$$

in the region $x \leq x_{\mathrm{m}}$. Substituting this into equation (8), we finally obtain

$$
\Sigma(x)=\Sigma_{\infty} \exp \left(-\frac{1}{2}\left(\frac{x}{h}-\frac{5}{4} \frac{x_{\mathrm{m}}}{h}\right)^{2}+\frac{1}{32}\left(\frac{x_{\mathrm{m}}}{h}\right)^{2}-\left(\frac{x_{\mathrm{m}}}{\ell}\right)^{-3}\right) \equiv \Sigma_{\mathrm{R}},
$$

for $x \leq x_{\mathrm{m}}$.

In summary, the equilibrium profile of surface density is given as

$$
\Sigma(x)= \begin{cases}\Sigma_{\mathrm{R}}(x) & \text { for } x \leq x_{\mathrm{m}}, \\ \Sigma_{\mathrm{vis}}(x) & \text { for } x \geq x_{\mathrm{m}}\end{cases}
$$

An example of the density profile is shown in Fig,1.

As mentioned above, we have assumed $v_{y}=-(3 / 2) \Omega_{\mathrm{p}} x$ in deriving equation (44). However, based on equation (9), the velocity at $x=x_{\mathrm{m}}$ is

$$
v_{y, \mathrm{~m}}=-\frac{11}{8} \Omega_{\mathrm{p}} x_{\mathrm{m}}
$$

This means that the maximum deviation from the Keplerian shear velocity, $-(3 / 2) \Omega_{\mathrm{p}} x$, is less than $10 \%$ of the absolute value. Hence the assumption of the Keplerian shear velocity is reasonable for $x \geq x_{\mathrm{m}}$. 
Finally we briefly describe the dependence of the surface density on the parameters. Figure 2 shows the surface density as a function of $x / r_{\mathrm{p}}$ for several different values of planetary mass $M_{\mathrm{p}}(\mathrm{a})$, viscosity $\nu$ (b), and scale height $h(\mathrm{c})$. Figures $2 \mathrm{a}$ and $2 \mathrm{~b}$ show the gap becomes deeper and wider with increasing planetary mass and decreasing viscosity. The width and depth of the gap are determined by competition between gravitational scattering by the planet and viscous diffusion of the disk gas. The gap becomes wider and deeper when planet's gravity is stronger or viscous diffusion is less efficient. From a mathematical point of view, this is because both $\Sigma_{\text {vis }}$ and $\Sigma_{\mathrm{R}}$ depends on $M_{\mathrm{p}}$ and $\nu$ in the form of $\left(M_{\mathrm{p}}^{2} / \nu\right)^{1 / 3}$ through $\ell$. As for the dependence of $\Sigma$ on $h$ (see Fig. 2k), all the curves are independent of $h$ for $x \geq x_{\mathrm{m}}$, where $\Sigma=\Sigma_{\text {vis }}$ which is independent of $h$ (see eq. [4]). In other words, surface density in $x \geq x_{\mathrm{m}}$ is determined by the balance between viscous torque and gravitational torque from the planet and has nothing to do with pressure. On the other hand, difference in surface density appears at $x<x_{\mathrm{m}}$, where $\Sigma$ is determined by the Rayleigh condition (see eq. 13]) and thus depends on all of $h, \nu$, and $M_{\mathrm{p}}$ through $x_{\mathrm{m}}$.

\section{Gas accretion rate onto planets}

In this study, we describe the gas accretion rate onto the planet, $\dot{M}_{\mathrm{p}}$. Through a series of local high-resolution hydrodynamic simulations of the gas accretion flow onto giant planets in protoplanetary disks, TW02 obtained an empirical relation between accretion rate normalized by unperturbed surface density and $h / r_{\mathrm{H}}$, which is the only one parameter of the local system (see eq.[18] of TW02). Furthermore, TW02 demonstrated that only the gas in the two bands at $|x| \sim 2 r_{\mathrm{H}}$ accretes onto the planet. Thus, writing equation (18) of TW02 in the explicit form, for $0.5 \leq h / r_{\mathrm{H}} \leq 1.8$, we have

$$
\dot{M}_{\mathrm{p}}=\dot{A} \Sigma_{\mathrm{acc}}
$$

where

$$
\dot{A} \simeq 0.29\left(\frac{h}{r_{\mathrm{p}}}\right)^{-2}\left(\frac{M_{\mathrm{p}}}{M_{*}}\right)^{4 / 3} r_{\mathrm{p}}^{2} \Omega_{\mathrm{p}},
$$

which is the area, the gas in which is to be accreted onto the planet per unit time (hereafter the accretion area), and $\Sigma_{\text {acc }}=\Sigma\left(2 r_{\mathrm{H}}\right)$.

The reason why small $h$ or large $M_{\mathrm{p}}$ yields high $\dot{M}_{\mathrm{p}}$ is explained as follows. Disk gas loses its energy by passing through spiral shocks around the planet and consequently accretes onto the planet. Small $h$ (i.e., small sound velocity) or large $M_{\mathrm{p}}$ (i.e., strong perturbation on gas motion) yields a large Mach number of the disk gas, which results in strong shocks. In addition, an increase in $M_{\mathrm{p}}$ expands the planetary feeding zone, resulting in large $\dot{M}_{\mathrm{p}}$. 
Note that the position of the accretion band, $x_{\mathrm{acc}}$, is located at $\sim 2 r_{\mathrm{H}}$ in the highestmass case of TW02 (i.e., $\tilde{C}_{\text {iso }}=0.5$ in their notation; see Fig. 7 of TW02 where $x_{\text {acc }}$ is shown to depend slightly on $h / r_{\mathrm{H}}$ ). When $\tilde{C}_{\text {iso }}<0.5$, a gap exists around the planetary orbit. In such situations, appropriate choice of $x_{\text {acc }}$ is crucial in determining $\dot{M}_{\mathrm{p}}$ because a small difference in $x_{\text {acc }}$ makes a large difference in $\Sigma_{\text {acc }}$ and thus $\dot{M}_{\mathrm{p}}$, while choice of $x_{\text {acc }}$ has little influence on $\dot{M}_{\mathrm{p}}$ in low-mass cases. That is why we adopt the value of $x_{\text {acc }}\left(=2 r_{\mathrm{H}}\right)$ for the highest-mass case of TW02.

Note also that, as shown by D'Angelo, Kley, \& Henning (2003), the gas accretion rate obtained by two-dimensional simulations are usually higher than that obtained by threedimensional simulations. Thus the accretion rate given by equation (16) could be overestimated. We will discuss this issue in $\$ 4.4$.

\section{Evolution}

In this section we show the evolution of the growth rate and mass of an accreting planet. To gain a proper understanding of the basic behavior of the planetary accretion, we first explore two simple cases with no disk dissipation (i.e. constant $\Sigma_{\infty}$ ) and with exponentiallydecreasing $\Sigma_{\infty}$ in $\$ \$ 4.1$ and 4.2 , respectively. Then, we investigate the accretion of a giant planet embedded in a viscously evolving protoplanetary disk in $\$ 4.3$.

The numerical procedure is as follows. Except in \$4.1, we first calculate the unperturbed surface density, $\Sigma_{\infty}$, from equation (21) in $\$ 4.2$ or from equation (26) in $\$ 4.3$. For given values of the parameters, $h / r_{\mathrm{p}}$ and $\nu /\left(r_{\mathrm{p}}^{2} \Omega_{\mathrm{p}}\right)$, we then calculate $\Sigma_{\text {acc }}$ and $\dot{A}$, using equation (4) if $x_{\text {acc }} \geq x_{\mathrm{m}}$ or equation (13) if $x_{\mathrm{acc}} \leq x_{\mathrm{m}}$. Finally we integrate $\dot{M}_{p}$ with respect to time using $\Sigma_{\text {acc }}$ and $\dot{A}$ (see eq. [16]) to obtain the time evolution of the planetary mass.

\subsection{Case without disk-dissipation}

We first show the evolution of the planetary mass without global disk dissipation. In this case the input parameters are $\Sigma_{\infty} r_{\mathrm{p}}^{2} / M_{*}, h / r_{\mathrm{p}}$, and $\nu /\left(r_{\mathrm{p}}^{2} \Omega_{\mathrm{p}}\right)$.

\subsubsection{General Properties}

Figure 3 shows the evolution of the planetary mass and the gas accretion rate for several values of the three parameters. Without global disk depletion, the only way to suppress gas 
accretion is opening a deep gap around the planetary orbit by the planet itself. As seen in Fig. 3, the evolution can be divided into two phases. The first phase is growth without a gap (pre-gap phase), while the second phase is growth with a deep gap (post-gap phase). Properties of the evolution in both phases can be explained in analytical ways below.

In the pre-gap phase, $\dot{M}_{\mathrm{p}}$ is almost proportional to $M_{\mathrm{p}}^{4 / 3}$ (see Figs. 3 -(c), (f), (i)). In this phase, the planetary mass is insufficient to open a gap, so that $\Sigma_{\text {acc }} \simeq \Sigma_{\infty}$. Then we can easily integrate equation (16) with equation (17):

$$
\dot{M}_{\mathrm{p}}=\frac{S \Sigma_{\infty} r_{\mathrm{p}}^{2} \Omega_{\mathrm{p}}\left(\frac{h}{r_{\mathrm{p}}}\right)^{-2}}{\left[\left(\frac{M_{\mathrm{p}, \text { init }}}{M_{*}}\right)^{-1 / 3}-\frac{S}{3}\left(\frac{\Sigma_{\infty}}{M_{*} / r_{\mathrm{p}}^{2}}\right)\left(\frac{h}{r_{\mathrm{p}}}\right)^{-2}\left(\frac{t}{\Omega_{\mathrm{p}}^{-1}}\right)\right]^{4}}
$$

where $S$ corresponds to 0.29 in equation (17) and $M_{\mathrm{p} \text {,init }}$ is the initial mass of the planet that corresponds to the mass beyond which the gas accretion is limited by disk-gas supply. This equation implies that if $\Sigma_{\text {acc }}$ is constant, the gas accretion rate diverges at a time

$$
\begin{aligned}
\tau_{\text {div }} & =\frac{3}{S}\left(\frac{M_{\mathrm{p}, \text { init }}}{M_{*}}\right)^{-1 / 3}\left(\frac{\Sigma_{\infty}}{M_{*} / r_{\mathrm{p}}^{2}}\right)^{-1}\left(\frac{h}{r_{\mathrm{p}}}\right)^{2} \Omega_{\mathrm{p}}^{-1} \\
& =4.8 \times 10^{4}\left(\frac{M_{\mathrm{p}, \text { init }}}{10^{-5} M_{*}}\right)^{-1 / 3}\left(\frac{\Sigma_{\infty}}{10^{-5} M_{*} / r_{\mathrm{p}}^{2}}\right)^{-1}\left(\frac{h}{10^{-1.5} r_{\mathrm{p}}}\right)^{2} \Omega_{\mathrm{p}}^{-1}
\end{aligned}
$$

$\tau_{\text {div }}$ corresponds to the end of the pre-gap phase.

In the post-gap phase, $\dot{M}_{\mathrm{p}}$ decreases with $M_{\mathrm{p}}$ in an exponential fashion (see Figs. 3 (c),(f),(i)). Such a steep decrease in $\dot{M}_{\mathrm{p}}$ is due to a steep decrease in $\Sigma_{\text {acc }}$ with respect to $M_{\mathrm{p}}$ via $\ell, x_{\mathrm{m}}$, and $r_{\mathrm{H}}$ (see eqs. [4] and [13]). We can derive approximate expressions for the accretion rate in this phase, which shows $\dot{M}_{\mathrm{p}}$ is inversely proportional to time as shown in Figs. 3(a)-(c) (see appendix for the detail).

\subsubsection{Dependence on the parameters}

We now see the dependence of the evolution of planetary mass on the unperturbed surface density, $\Sigma_{\infty}$ (Figs. 3-(a),(b),(c)). The evolution timescale is inversely proportional to $\Sigma_{\infty}$ (e.g., Fig. 3-(b)). This is simply because $\Sigma_{\text {acc }}$ is proportional to $\Sigma_{\infty}$, whereas $\dot{A}$ is independent of $\Sigma_{\infty}$. The peak value of $\dot{M}_{\mathrm{p}}$ is thus proportional to $\Sigma_{\infty}$ accordingly (e.g., Fig. $3(\mathrm{c}))$. 
The dependence on scale height, $h$, is shown in Figs. 3-(d),(e),(f). In the pre-gap phase, $\dot{M}_{\mathrm{p}}$ is simply proportional to $h^{-2}$ and the evolution timescale is thus $\propto h^{2}$. This is because the accretion area $\dot{A} \propto h^{-2}$ (see eq. [17]) in the pre-gap phase where $\Sigma_{\text {acc }}=\Sigma_{\infty}$. Even in the post-gap phase, the dependence of $\dot{M}_{\mathrm{p}}$ on $h$ is the same as that in the pre-gap phase if $h$ (i.e., $\left.x_{\mathrm{m}}\right)$ is small $(h / r \lesssim 0.03)$. As long as $h$ is so small that $x_{\mathrm{m}} \leq 2 r_{\mathrm{H}}, \Sigma_{\text {acc }}$ is determined by $\Sigma_{\mathrm{vis}}$ and is thus independent of $h$ (see eqs. (4) and [14). When $h$ is so large that $x_{\mathrm{m}} \geq 2 r_{\mathrm{H}}$, $\Sigma_{\text {acc }}=\Sigma_{\mathrm{R}}$, which depends on $h$. Hence $\Sigma_{\text {acc }}$ increases with $h$ for large $h$. The transition between the low- $h$ and high- $h$ cases takes place when $x_{\mathrm{m}}=2 r_{\mathrm{H}}$, namely, from equation (11):

$$
h=0.037 r_{\mathrm{p}}\left(\frac{M_{\mathrm{p}}}{10^{-3} M_{*}}\right)^{-1 / 6}\left(\frac{\nu}{10^{-5} r_{\mathrm{p}}^{2} \Omega_{\mathrm{p}}}\right)^{1 / 2} .
$$

The dependence on viscosity, $\nu$, is shown in Fig 3 (g) $(\mathrm{h})(\mathrm{i})$. In the pre-gap phase, the evolution of $\dot{M}_{\mathrm{p}}$ is almost the same for different values of $\nu$, because $\Sigma_{\text {acc }}$ is almost constant $\left(\simeq \Sigma_{\infty}\right)$ in the pre-gap phase and $\dot{A}$ is independent of $\nu$. Thus the timing when $\dot{M}_{\text {p }}$ reaches at the peak value does not depend on $\nu$ either (see eq.19]). In the post-gap phase, $\dot{M}_{\mathrm{p}}$ at a given $t$ increases with $\nu$. In appendix $\mathbb{B}$, we have derived approximate solutions for $\dot{M}_{\mathrm{p}}$ in the post-gap phase. From equations (B2) and (B3), one finds that $M_{\mathrm{p}} \propto \nu$ in high- $\nu$ cases (i.e., $x_{\text {acc }}>x_{\mathrm{m}}$ ). In low- $\nu$ cases (i.e., $\left.x_{\mathrm{acc}}<x_{\mathrm{m}}\right), \dot{M}_{\mathrm{p}}$ also increases with $\nu$, but the dependence is rather weak (see eqs. [B7] and [B8). Also, the accretion rate is inversely proportional to time (see eqs. [B3] or [B8]), so that the maximum accretion rate can be roughly estimated by eqs. $\mathrm{B} 3$ ] or $\left[\mathrm{B} 8\right.$, at $t=\tau_{\text {div }}$.

\subsection{Case with exponential disk dissipation}

Next we examine the evolution of the planetary mass in a simple case where the disk surface density decreases in an exponential fashion with a time constant of $\tau_{\text {dep }}$ :

$$
\Sigma_{\infty}=\Sigma_{\infty, \text { init }} \exp \left(-\frac{t}{\tau_{\mathrm{dep}}}\right),
$$

where $\Sigma_{\infty, \text { init }}$ is the initial surface density at infinity.

Figure 4 shows the evolution of $M_{\mathrm{p}}$ and $\dot{M}_{\mathrm{p}}$ for $\tau_{\text {dep }}=10^{6} \Omega_{\mathrm{p}}^{-1}$ and three values of $\Sigma_{\infty \text {,init }}$. In the high- $\Sigma_{\infty, \text { init }}$ case $\left(\Sigma_{\infty, \text { init }} /\left(M_{*} r_{\mathrm{p}}^{-2}\right)=10^{-4.5}\right), \dot{M}_{\mathrm{p}}$ increases with time to reach a peak at $t \sim 1.5 \times 10^{5} \Omega_{\mathrm{p}}^{-1}$ and then decreases with time, which is similar to the evolution without disk dissipation shown in 44.1. This is because $\tau_{\text {div }}<\tau_{\text {dep }}$ in this case. In the low- $\Sigma_{\infty, \text { init }}$ case $\left(\Sigma_{\infty, \text { init }} /\left(M_{*} r_{\mathrm{p}}^{-2}\right)=10^{-6.5}\right)$, on the other hand, $\dot{M}_{\mathrm{p}}$ decreases without experiencing a significant increase that occurs in the high- $\Sigma_{\infty, \text { init }}$ case. This is because $\tau_{\text {div }}>\tau_{\text {dep }}$ in this case 
$\left(\tau_{\text {div }} \propto \Sigma_{\infty, \text { init }}^{-1}\right.$; see eq. [19]). Because of such different evolution of $\dot{M}_{\mathrm{p}}$, the mass evolution also differs between high- and low- $\Sigma_{\infty, \text { init }}$ cases (see Fig. 4b): The planet captures a significant amount of gas to be a Jupiter-like planet in the high- $\Sigma_{\infty}$,init case, whereas the planet captures only a small amount of gas to be a Neptune-like planet in the low- $\Sigma_{\infty}$,init case. The boundary between the two regimes is determined by the condition $\tau_{\text {dep }}=\tau_{\text {div }}$, namely, from equation (19),

$$
\frac{\Sigma_{\infty, \text { init }}}{M_{*} / r_{\mathrm{p}}^{2}}=\frac{3}{S}\left(\frac{M_{\mathrm{p}, \text { init }}}{M_{*}}\right)^{-1 / 3}\left(\frac{h}{r_{\mathrm{p}}}\right)^{2}\left(\frac{\tau_{\mathrm{dep}}}{\Omega_{\mathrm{p}}^{-1}}\right)^{-1}
$$

Figure 5 shows the final mass defined by

$$
M_{\text {final }} \equiv \int_{0}^{\infty} \dot{M}_{\mathrm{p}} d t
$$

as a function of a quantity $\zeta$ defined by

$$
\zeta \equiv\left(\frac{\Sigma_{\infty, \text { init }}}{M_{*} / r_{\mathrm{p}}^{2}}\right)\left(\frac{\tau_{\mathrm{dep}}}{\Omega_{\mathrm{p}}^{-1}}\right) .
$$

The dependence of $M_{\text {final }}$ on $\tau_{\text {dep }}$ is the same as that on $\Sigma_{\infty \text {,init }}$ because the ratio of $\tau_{\text {div }}$ $\left(\propto \Sigma_{\infty, \text { init }}^{-1}\right)$ to $\tau_{\text {dep }}$ determines the evolution. Figure 5 illustrates that when $\zeta$ is small $(\lesssim 1)$, the final mass is almost the same as the initial mass. In this regime, the planet captures only a tiny amount of gas and becomes a Neptune-like planet. When $\zeta \geq 10$, on the other hand, the planet captures substantial gas to be a gas giant planet like Jupiter and the final mass does not depend on the initial planetary mass. This is because the planet becomes large enough to form a gap and the evolution slows down significantly after gap formation; the condition for gap opening is determined by the planetary mass. The transition occurs when $\tau_{\text {div }}=\tau_{\text {dep }}$, namely, from equation (19),

$$
\zeta_{\mathrm{t}} \simeq 4.8\left(\frac{M_{\mathrm{p}, \mathrm{init}}}{10^{-5} M_{*}}\right)^{-1 / 3}\left(\frac{h}{0.1 r_{\mathrm{p}}}\right)^{2} .
$$

Figure 6] shows that the final mass as a function of $\zeta$ and $\nu$ in the cases with $h / r=0.1$ and $h / r=0.032$. A portion characterized by a steep gradient is illustrated to shift to the left with decreasing $h / r_{\mathrm{p}}\left(\zeta_{\mathrm{t}} \sim 5\right.$ for $h / r_{\mathrm{p}}=0.1$, while $\zeta_{\mathrm{t}} \sim 0.1$ for $h / r_{\mathrm{p}}=0.032$, see eq. 225]). On the left side to the portion (low- $\zeta$ case), the final mass is almost equal to the initial mass and thus almost independent of viscosity. On the right side, the final mass increases with viscosity. This is because the growth is limited by opening a deep gap; the mass for gap opening depends on viscosity. 


\subsection{Case with global disk evolution}

\subsection{1. the model}

We have so far neglected global evolution of the disk, although we examined a simple case with an exponential depletion of the disk gas in $\$ 4.2$. In reality, the unperturbed surface density, $\Sigma_{\infty}$, changes because of global disk evolution. In this section, we examine the effects of global disk evolution on planetary growth.

Because a detailed description of disk evolution is beyond the scope of this paper, we assume that the disk surface density changes with time in such a way

$$
\Sigma_{\infty}=\Sigma_{\mathrm{ss}}(r, t) \exp \left(-\frac{t}{\tau_{\text {dep }}}\right)
$$

instead of equation (21): $\Sigma_{\mathrm{ss}}$ represents a change in the surface density due to viscous diffusion of the disk gas and $e^{-t / \tau_{\mathrm{dep}}}$ is introduced to mimic photoevaporation of the disk. For $\Sigma_{\mathrm{ss}}$, we adopt the self-similar solution with $\alpha$ prescription for disk viscosity given by (Hartmann et al. 1998)

$$
\Sigma_{\mathrm{ss}}(r, t)=\frac{M_{\text {disk }}}{2 \pi R_{\text {out }}^{2}}\left(\frac{r}{R_{\text {out }}}\right)^{-1} \tilde{\tau}_{\mathrm{ss}}^{-3 / 2} \exp \left(-\frac{r / R_{\text {out }}}{\tilde{\tau}_{\mathrm{ss}}}\right),
$$

where $M_{\text {disk }}$ is the initial disk mass and $R_{\text {out }}$ is the initial disk size; $\tilde{\tau}_{\text {ss }}$ is defined as

$$
\tilde{\tau}_{\mathrm{ss}}=\frac{t}{\tau_{\mathrm{vis}}}+1
$$

with a typical timescale of global viscous evolution

$$
\begin{aligned}
\tau_{\text {vis }} & \equiv \frac{R_{\text {out }}^{2}}{3 \nu_{\text {out }}} \\
& =5.3 \times 10^{5}\left(\frac{\alpha}{0.01}\right)^{-1}\left(\frac{h_{1 \mathrm{AU}}}{10^{-1.5} \mathrm{AU}}\right)^{-2}\left(\frac{R_{\text {out }}}{100 \mathrm{AU}}\right) \mathrm{yr},
\end{aligned}
$$

where $\nu_{\text {out }}$ is viscous coefficient of the disk gas at $r=R_{\text {out }}$. In deriving equation (27), we have assumed a temperature distribution, $T \propto r^{-1 / 2}$, which results in $\nu \propto r$.

Using the equations above, we calculate the local gas accretion rate onto the planet, denoted hereafter by $\dot{M}_{\mathrm{p} \text {,local }}$, in the same way as we did in $\$ 4.2$. However, when $\dot{M}_{\mathrm{p}, \text { local }}$ is larger than the radial mass flux due to viscous diffusion in the disk, the latter limits the planetary growth. The mass flux through a ring with radius $r$ is given by

$$
\begin{aligned}
\dot{M}_{\text {disk }}(r, t) & =2 \pi r \Sigma_{\mathrm{ss}}(r, t) v_{r} \\
& =\frac{M_{\text {disk }}}{\tau_{\text {vis }}}\left(\frac{1}{2}-\frac{r}{\tilde{\tau}_{\mathrm{ss}} R_{\text {out }}}\right) \tilde{\tau}_{\mathrm{ss}}^{-3 / 2} \exp \left(-\frac{r}{\tilde{\tau}_{\mathrm{ss}} R_{\text {out }}}\right),
\end{aligned}
$$


with the radial drift velocity of the diffusing gas in a Keplerian disk

$$
v_{r}=-\frac{3 \nu}{r}\left(\frac{\partial \ln (\Sigma \nu)}{\partial \ln r}+\frac{1}{2}\right)
$$

Thus we calculate the gas accretion rate onto the planet in such a way

$$
\dot{M}_{\mathrm{p}}= \begin{cases}\dot{M}_{\mathrm{p}, \text { local }} & \text { if } \dot{M}_{\mathrm{p}, \text { local }}<\dot{M}_{\text {disk }} \\ \dot{M}_{\text {disk }} & \text { if } \dot{M}_{\mathrm{p}, \text { local }}>\dot{M}_{\text {disk }}\end{cases}
$$

There are several studies that modeled gas accretion rate onto gas giant planets. Guillot \& Hueso (2006) used a formula in which the gas accretion rate onto the planet is 0.3 times the radial mass flux of the disk gas due to global viscous diffusion. However their formula is an empirical one and applies in a limited situation. Lubow, Seibert, \& Artymowicz (1999) carried out a series of hydrodynamic simulations to obtain gas accretion rate onto planets. Their simulations demonstrated that the planetary accretion rate can be larger than the diffusion flux because of gradients imposed by the gap. However, what they found is probably a transient phenomenon that occurs on a timescale much shorter than the viscous timescale. Veras \& Armitage (2004) used an approximate formula based on hydrodynamic simulations done by Lubow, Seibert, \& Artymowicz (1999) and D'Angelo, Henning, \& Kley (2002).

\subsection{2. dependence on the disk parameters}

Figure 7 is a similar figure to Fig. 6 and shows the final mass of the planet, $M_{\text {final }}$, as a function of the initial surface density, $\Sigma_{\infty}$,init, and viscosity, $\nu$, in the case where $h / r=0.1$ (left panel) and 0.032 (right panel), $R_{\text {out }}=10 r_{p}$, and $\tau_{\text {dep }}=10^{6} \Omega_{\mathrm{p}}^{-1}$. The value of $\tau_{\text {dep }}$ corresponds to $\zeta=10^{6}\left(\Sigma_{\infty \text {,init }} /\left(M_{*} r_{\mathrm{p}}^{-2}\right)\right)$, so that the ranges of this figure are the same as those of Figure 6 for the horizontal axis as well as the vertical axis.

Since we have adopted the self-similar solution for the surface density evolution of the global disk, there appear two additional limits to the final mass. One arises from the appearance of the viscous timescale $\tau_{\text {vis }}$, in addition to the timescale of exponential decay $\tau_{\text {dep }}$. When $\tau_{\text {vis }}<\tau_{\text {dep }}$, the effective disk lifetime is $\tau_{\text {vis }}$. Thus, in order for planets to be massive, the growth timescale $\tau_{\text {div }}$ should be shorter than $\tau_{\text {vis }}$, so that the additional limit is given by $\tau_{\mathrm{vis}}=\tau_{\mathrm{div}}:$

$$
\frac{\nu}{r_{\mathrm{p}}^{2} \Omega_{\mathrm{p}}} \simeq 7 \times 10^{-5}\left(\frac{M_{\mathrm{p}, \text { init }}}{10^{-5} M_{*}}\right)^{1 / 3}\left(\frac{h}{0.1 r_{\mathrm{p}}}\right)^{-2}\left(\frac{\Sigma_{\infty}}{10^{-4} M_{*} / r_{\mathrm{p}}^{2}}\right)\left(\frac{R_{\text {out }}}{10 r_{\mathrm{p}}}\right),
$$

which runs from the lower left to the upper right in Fig:7 because $\nu$ is linearly proportional to the surface density. As described above, the effective disk lifetime, $\tau_{\text {lifetime, }}$ is the shorter 
one of the two timescales, so that we here define

$$
\tau_{\text {lifetime }}= \begin{cases}\tau_{\text {dep }} & \text { if } \tau_{\text {dep }}<\tau_{\text {vis }} \\ \tau_{\text {vis }} & \text { if } \tau_{\text {dep }}>\tau_{\text {vis }}\end{cases}
$$

The other limit is the total mass of the disk. This limit does not appear in Fig,6(i.e., the case without global viscous evolution). This can be clearly seen in the case of $h / r=0.032$ on the right panel of Fig. 7 where contour lines are vertical. Note that the steep boundary (being equivalent to $\zeta=\zeta_{\mathrm{t}}$ ) characterized by $\tau_{\text {div }}=\tau_{\text {dep }}$ that is seen in Fig 6 is not found in Fig. 7. This is simply because the two limits described above are more severe than the condition of $\tau_{\text {div }}=\tau_{\text {dep }}$ in the cases shown in Figure 7. However, when $\tau_{\text {dep }}$ is smaller, the boundary determined by $\tau_{\text {div }}=\tau_{\text {dep }}$ moves rightward, so that the vertical steep boundary determined by $\tau_{\text {div }}=\tau_{\text {dep }}$ emerges even in the case with global disk evolution described in this subsection.

\subsection{3. classification by semi-major axis}

In Figure 7, the dependence of the final mass was shown for the normalized disk parameters. However, one might want to know its dependence on semi-major axis. We thus show the final mass as a function of semi-major axis in Figure 8 for a typical case with $\alpha=0.01$, $h / r=0.032$ at $1 \mathrm{AU}, \tau_{\text {dep }}=10^{6} \mathrm{yr}, R_{\text {out }}=100 \mathrm{AU}, M_{\mathrm{p}, \text { init }}=3.2 \times 10^{-5} M_{*}$, which corresponds to 10 Earth masses in the solar mass system, and $M_{\text {disk }}=0.013 M_{*}$, which yields a surface density roughly 0.1 times that of minimum mass solar nebula at $1 \mathrm{AU} 2$. In addition to the final mass given by equation (23), two kinds of the mass defined by

$$
M_{\mathrm{p}, \text { local }} \equiv \int_{0}^{\infty} \dot{M}_{\mathrm{p}, \text { local }} d t \quad \text { and } \quad M_{\mathrm{p}, \text { disk }} \equiv \int_{0}^{\infty} \dot{M}_{\text {disk }} d t
$$

are shown to clarify the limiting process for the final mass.

As shown in Figure 8, the final mass (solid line) is determined basically by the smaller of $M_{\mathrm{p} \text {,local }}$ (dotted line) and $M_{\mathrm{p}, \text { disk }}$ (dashed line), although the limiting process varies with time. Thus one finds three characteristic regions that are divided at the intersection of the two curves.

In the inner region $\left(r_{p} \lesssim 1 \mathrm{AU}\right), M_{\text {final }}$ is almost equal to $M_{\mathrm{p}, \text { local }}$ and is proportional to $r_{p}^{1 / 2}$. The duration of the pre-gap phase is shorter than the disk lifetime (i.e., $\tau_{\text {div }}<\tau_{\text {lifetime }}$ )

\footnotetext{
${ }^{2}$ This surface density is much lower than the minimum mass solar nebula model because the slope of the surface density is shallower and the disk size is larger.
} 
in this region. The gas accretion is thus suppressed because of gap opening. This region is hereafter called the gap-limiting region. In this situation the final mass can be roughly estimated by integrating $\dot{M}_{\mathrm{p}}$ from $\tau_{\text {div }}$ to $\tau_{\text {lifetime. }}$. Since $\tau_{\text {div }}<\tau_{\text {lifetime }}$ in the gap-limiting region for the values of the parameters used in drawing Fig. 8, $M_{\text {final }}$ is readily calculated as (see Appendix (B)

$$
\begin{aligned}
M_{\text {final }} & \simeq \int_{\tau_{\text {div }}}^{\tau_{\text {lifetime }}} \frac{M_{\text {trans }}}{t} d t \\
& =M_{\text {trans }} \log \left(\frac{\tau_{\text {lifetime }}}{\tau_{\text {div }}}\right) \equiv M_{\text {final,gap }}
\end{aligned}
$$

where $M_{\text {trans }}$, defined by equation (B2), is a planetary mass when $\Sigma_{\text {acc }}=\Sigma_{\infty} / e$ and $2 r_{\mathrm{H}} \leq x_{\mathrm{m}}$. Except for the weak dependence on the other parameters included in the log term, the final mass given by equation (36) is determined principally by $\nu$ and increases with $\nu$. This is because larger planetary mass is required for the planet to open a gap in the case of higher viscosity. Since $M_{\text {trans }} \propto \nu /\left(r_{p}^{2} \Omega_{p}\right)$ and $\nu \propto r_{p}$ in our disk model, $M_{\text {trans }} \propto \alpha r_{p}^{1 / 2}$, which is consistent with the result shown in Fig. 8.

In the intermediate region $\left(1 \mathrm{AU} \lesssim r_{p} \lesssim 100 \mathrm{AU}\right)$, Figure 8 illustrates

$$
M_{\text {final }} \simeq M_{\mathrm{p}, \mathrm{disk}}
$$

In the region, viscous diffusion of the disk gas limits the gas accretion onto the planet. This region is hereafter called the diffusion-limiting region. In this region, radial mass flux due to viscous diffusion is uniform where $r_{\mathrm{p}} \ll R_{\text {out }}$, so that the final mass is usually insensitive to $r_{\mathrm{p}}$. In the case with $\tau_{\text {vis }}<\tau_{\text {dep }}$, which is the case of Fig. 8 , most of the disk gas is transferred toward the central star by viscous diffusion, thus the final mass is roughly the disk mass $\left(\simeq 0.013 M_{*}\right)$. Hence we have

$$
M_{\text {final,diff }} \sim M_{\text {disk }} .
$$

On the other hand, when $\tau_{\text {vis }} \gg \tau_{\text {dep }}$, evaporation dominates viscous diffusion in disk dissipation. In this situation, the final mass at $r \ll R_{\text {out }}$ is given by

$$
\begin{aligned}
M_{\text {final,diff }} & \simeq \int_{0}^{\tau_{\text {dep }}}\left|\dot{M}_{\text {disk }}\right| d t \\
& \simeq \frac{1}{2}\left(\frac{\tau_{\text {dep }}}{\tau_{\text {vis }}}\right) M_{\text {disk }} .
\end{aligned}
$$

Note that, even when $\tau_{\mathrm{vis}} \gg \tau_{\mathrm{dep}}$, the gas accretion rate onto the planet is limited by viscous diffusion.

In the outer region $\left(r_{p} \gtrsim 100 \mathrm{AU}\right)$, the final mass suddenly decreases to $M_{\mathrm{p} \text {,init }}$. This is because the planetary growth finishes in the pre-gap phase because of the dissipation of the disk gas (i.e., $\tau_{\text {div }} \gtrsim \tau_{\text {lifetime }}$ ). We call this region the no-growth region. 
The positions of the boundaries between the three regions are given as follows. At the boundary between the gap-limiting region and the diffusion-limiting region (its position

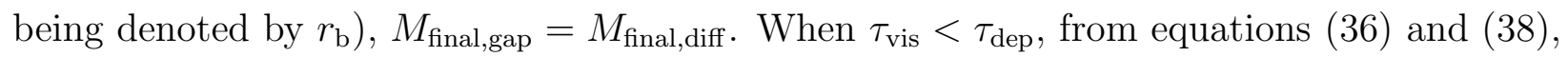
we obtain

$$
r_{\mathrm{b}} \simeq 5\left(\frac{\alpha}{10^{-2}}\right)^{-2}\left(\frac{h_{1 \mathrm{AU}}}{10^{-1.5} \mathrm{AU}}\right)^{-4}\left(\frac{M_{\text {disk }}}{10^{-2} M_{*}}\right)^{2}\left(\frac{\log \left(\tau_{\mathrm{vis}} / \tau_{\text {div }}\right)}{5}\right)^{-2} .
$$

Here we have assumed the log factor in equation (36) is constant. When $\tau_{\text {vis }}>\tau_{\text {dep }}$, from equations (36) and (39), we have

$$
r_{\mathrm{b}} \simeq 1\left(\frac{\alpha}{10^{-2}}\right)^{-2}\left(\frac{h_{1 \mathrm{AU}}}{10^{-1.5} r_{1 \mathrm{AU}}}\right)^{-4}\left(\frac{M_{\text {disk }}}{10^{-2} M_{*}}\right)^{2}\left(\frac{\tau_{\text {dep }}}{\tau_{\text {vis }}}\right)^{2}\left(\frac{\log \left(\tau_{\text {dep }} / \tau_{\text {div }}\right)}{5}\right)^{-2} .
$$

The location of the boundary between the diffusion-limiting region and the no-growth region is basically determined by $\tau_{\text {div }} \sim \tau_{\text {lifetime }}$ and is denoted by $r_{\mathrm{e}}$. When $\tau_{\text {vis }}<\tau_{\text {dep }}$, from $\tau_{\text {div }}=\tau_{\text {vis }}$, we obtain

$$
r_{\mathrm{e}} \simeq 70\left(\frac{M_{\mathrm{p}, \text { init }}}{10^{-5} M_{*}}\right)^{1 / 3}\left(\frac{\Sigma_{\infty, 1 \mathrm{AU}}}{10^{-5} M_{*} / 1 \mathrm{AU}^{2}}\right)\left(\frac{h_{1 \mathrm{AU}}}{10^{-1.5} \mathrm{AU}}\right)^{-4}\left(\frac{\alpha}{10^{-2}}\right)^{-1}\left(\frac{R_{\text {out }}}{100 \mathrm{AU}}\right) \mathrm{AU} .
$$

When $\tau_{\text {vis }}>\tau_{\text {dep }}$, from $\tau_{\text {div }}=\tau_{\text {dep }}$, we obtain

$$
r_{\mathrm{e}} \simeq 130\left(\frac{M_{\mathrm{p}, \text { init }}}{10^{-5} M_{*}}\right)^{1 / 3}\left(\frac{\Sigma_{\infty, 1 \mathrm{AU}}}{10^{-5} M_{*} / 1 \mathrm{AU}^{2}}\right)\left(\frac{h_{1 \mathrm{AU}}}{10^{-1.5 \mathrm{AU}}}\right)^{-2}\left(\frac{\tau_{\mathrm{dep}}}{10^{6} \mathrm{yr}}\right) \mathrm{AU} .
$$

Figure 9 shows the final mass for several different values of the disk parameters. The discussion above suffices to understand this figure. Note that there is a hollow at $\sim 50 \mathrm{AU}$ in the case where the value of $\alpha$ is lower by a factor of 10 than its nominal value (thick short-dashed line). This is, however, artificial. In the self-similar solution we have adopted, the direction of the radial gas flow due to viscous diffusion changes at $r=\left(R_{\text {out }} / 2\right) \tilde{\tau}_{\mathrm{ss}}$, which means that the mass flux is zero at the point, and the accretion rate onto the planet is also zero accordingly (see eq. 32]). Since $\tilde{\tau}_{\mathrm{ss}}$ increases with time (see eq. 28]), the point moves outward with time. When $\tau_{\text {dep }}>\tau_{\text {vis }}$, the point moves significantly with time and integration of mass flux at a particular point eliminates the effect. In contrast, when $\tau_{\text {dep }} \ll \tau_{\text {vis }}$, the point hardly changes before the exponential depletion; the planet around $r=R_{\text {out }} / 2$ thus cannot grow significantly.

Another important aspect is on the final mass in the gap-limiting region. We can see that the $r_{\mathrm{p}}$ dependence of $M_{\text {final }}$ agrees with that of the mass given by the viscous condition for gap opening (Lin \& Papaloizou 1979, 1993):

$$
\begin{aligned}
M_{\mathrm{p}, \mathrm{vis}} & =40\left(\frac{\nu}{r^{2} \Omega}\right) M_{*} \\
& =4 \times 10^{-4}\left(\frac{\alpha}{10^{-2}}\right)\left(\frac{r}{1 \mathrm{AU}}\right)^{1 / 2} M_{*} .
\end{aligned}
$$


The dependence also agrees with that of $M_{\text {trans }}$, which is the mass when $\Sigma_{\text {acc }}$ is $(1 / e) \Sigma_{\infty}$ in the case of $2 r_{\mathrm{H}} \geq x_{\mathrm{m}}$ (see eq. $\mathrm{B} 2$ ), and re-written as

$$
M_{\mathrm{trans}} \simeq 9 \times 10^{-4}\left(\frac{\alpha}{10^{-2}}\right)\left(\frac{r}{1 \mathrm{AU}}\right)^{1 / 2} M_{*} .
$$

Both of the two masses indicate gap-forming masses and they agree with each other within a factor of about 2. However the final mass shown in our model is larger by a factor of 10 than $M_{\mathrm{p} \text {,vis }}$ or $M_{\text {trans }}$. This is because the two masses correspond to the masses at which a gap is about to form and does not mean the masses at which growth stops. A further increase in $M_{\mathrm{p}}$ after reaching $M_{\mathrm{p}, \text { vis }}$ or $M_{\text {trans }}$ is reflected in the log term in equation (36).

\subsection{Remarks on the uncertainties in the model}

We have assumed that the distribution of the disk gas around the planetary orbit (i.e., the surface density) is always in the equilibrium state that is determined by the balance between viscous stress and gravitational scattering by the planet. However, for the equilibrium state to be achieved, non-equilibrium distributions must be relaxed by diffusion. The diffusion timescale, $\tau_{\text {vis,local }}$, is estimated by the gap width $\left(\sim 2 \times 2 r_{\mathrm{H}}\right)$ divided by the viscosity as

$$
\tau_{\text {vis }, \text { local }}=7.7 \times 10^{3}\left(\frac{M_{\mathrm{p}}}{10^{-3} M_{*}}\right)^{2 / 3}\left(\frac{\nu}{10^{-5} r_{\mathrm{p}}^{2} \Omega_{\mathrm{p}}}\right)^{-1} \Omega_{\mathrm{p}}^{-1} .
$$

This timescale should be compared with the typical growth timescale (i.e., $\tau_{\text {div }}$; see eq. [19]). The comparison indicates that $\tau_{\text {vis,local }}<\tau_{\text {div }}$ in most of the cases presented in this paper. Hence the assumption is appropriate unless quite low viscosities (yielding large $\tau_{\text {vis,local }}$ ) or high surface densities (yielding small $\tau_{\text {div }}$ ) are considered.

The empirical formula for the accretion rate based on local two-dimensional isothermal hydrodynamic simulations (TW02) can be different from that derived based on more realistic simulations including, for example, three-dimensional accretion flow (D'Angelo, Kley, \& Henning 2003; Bate et al. 2003), non-isothermal equation of state (TW02), and magnetic field (Machida, Inutsuka, \& 2006). The modification would change the form of $\tau_{\text {div }}$, which would yield quantitatively different results. However, even in that case, following our prescription given in this paper, one can easily calculate the mass evolution of planets in a similar way. The formula given by TW02 can be considered as the highest limit; thus, for example, $r_{\mathrm{e}}$ is expected to shift inward if more realistic models for the accretion rate are used. We compare the accretion rate eq. 16] with those obtained by global simulations (Klev 1999; Lubow \& D'Angelo 2006). Although the value of our accretion rate are usually larger (by up to a factor of 10) than those given by the simulations, the dependence on viscosity and planetary mass is consistent. 
We have not taken into account a limit in terms of geometry of the accretion flow, namely a within which equation (17) is applicable. We can make a simple estimate of the maximum accretion rate if we assume all the gas within $0 \leq x \leq 2 \sqrt{3} r_{\mathrm{H}}$ in $y>0$ and $-2 \sqrt{3} r_{\mathrm{H}} \leq x \leq 0$ in $y<0$ accrete to the planet, where $x=2 \sqrt{3} r_{\mathrm{H}}$ at $y \gg r_{\mathrm{H}}$ is the point where potential energy on the rotating frame is the same as that at the Lagrange points L1 or L2. In this case, the maximum accretion rate (normalized by surface density) is given by $3^{1 / 3} 6 r_{\mathrm{p}}^{2} \Omega_{\mathrm{p}}\left(M_{\mathrm{p}} / M_{*}\right)^{2 / 3}$ and thus $\dot{A}$ (eq. [17) becomes larger than the maximum value when

$$
M_{\mathrm{p}}>5.6 \times 10^{-3} M_{*}\left(\frac{h}{0.032 r_{\mathrm{p}}}\right)^{3} \equiv M_{\mathrm{p}, \mathrm{crit}} .
$$

Although planetary mass can be larger than $M_{\mathrm{p}, \mathrm{crit}}$ depending on the parameters, such a massive planet should already has a deep gap, so that the accretion rate is reduced greatly when the planetary mass reaches $M_{\mathrm{p}, \mathrm{crit}}$. Consequently the geometric effect has no significant influence on the final mass. Note that the discussion here is based on an approximation that streamlines can be well described by particle motions on the framework of the restricted three-body problem. Since this approximation is not valid when $r_{\mathrm{H}}<h$ (Masset, D'Angelo, and Kley 2006), it is not applicable for small mass planet's cases. However, we consider the upper limit of applicable mass, where the planet mass is, in most cases, large enough to satisfy $r_{\mathrm{H}}>h$, so that the approximation is valid for the situations that we consider here.

In this paper, we have put off the issue of planetary migration. Since we consider a phase in which a gap exists around the planet, we may have to consider the type II migration (e.g. Ward 1997; Ward and Harn 2000), especially in the gap-limiting region (e.g., $r_{p}<1$ AU in Figure 8). The deep gap created by the planet blocks the accretion flow toward the central star. It follows that the planet is pushed inward by the gas exterior to the planet's orbit. Inclusion of the effect of planetary migration is our future work.

\section{Summary}

To gain a systematic understanding of the final masses of gas giant planets, we have simulated the long-term accretion of gas giant planets after the onset of the supercritical gas accretion in a variety of situations, depending on four disk parameters such as disk mass, viscosity, scale height, and semi-major axis. To do so, we have made a semi-analytical model to simulate the mass evolution, which enabled us to study the final mass of gas giant planets for extensive ranges of all the parameters.

We have first made a 1D analytical model of the equilibrium surface density profile 
around a protoplanet, from consideration of the balance of torque and the dynamical stability (\$2). Combining the surface density profile with an empirical formula for gas accretion rate that was obtained on the basis of hydrodynamic simulations by TW02, we have obtained a formula for gas accretion rate as a function of planetary mass, viscosity, scale height, and unperturbed surface density (\$3). We have then integrated the gas accretion rate numerically with respect to time to simulate the long-term accretion of gas giant planets $(\S 4)$. To understand the basic behavior of the planetary accretion, we have explored two simple cases with no disk dissipation (\$4.1) and with exponentially-decreasing surface density (\$4.2). Finally, we have simulated the long-term accretion of gas giant planets embedded in a viscously evolving and evaporating disk to obtain the final mass of gas giant planets as a function of semi-major axis (\$4.3). We have consequently found the following three different regions depending on limiting processes on the final mass.

In the inner region $\left(r_{\mathrm{p}} \lesssim r_{\mathrm{b}}\right.$; see eqs. 40] \& 41]), the planet grows quickly to form a deep gap to suppress the gas accretion from the disk by itself within the disk lifetime (gaplimiting region). We have found the final mass in this region is roughly 10 times larger than that determined by the viscous condition for gap opening (Lin \& Papaloizou 1993). This is because the condition for gap opening only expresses the condition when a gap begins to form, and is by no means equivalent to the condition that the growth is terminated.

In the intermediate region $\left(r_{\mathrm{b}} \lesssim r_{\mathrm{p}} \lesssim r_{\mathrm{e}}\right.$; see eqs. 42] \& [43]), radial transfer of the disk gas toward the planetary orbit limits the gas accretion before the planet opens a deep gap; the final mass is thus limited by viscous diffusion of the disk (diffusion-limiting region). We have found that when the evaporation timescale $\tau_{\text {dep }}$ is shorter than the viscous-diffusion timescale $\tau_{\text {vis }}$, the relationship between the final mass $M_{\text {final }}$ and the disk mass $M_{\text {disk }}$ is given by $M_{\text {final }} \sim(1 / 2)\left(\tau_{\text {dep }} / \tau_{\text {vis }}\right) M_{\text {disk }}$, whereas $M_{\text {final }} \sim M_{\text {disk }}$ when $\tau_{\text {dep }}>\tau_{\text {vis }}$.

In the outer region $\left(r \gtrsim r_{\mathrm{e}}\right)$, the planet captures only a tiny amount of gas by the time the disk gas completely dissipates (no-growth region). Saturn and possibly Uranus/Neptune are likely to have experienced the situation.

In this study, we have gained a clear understanding of the final masses of gas giant planets, deriving analytical expressions for them in three characteristic regions (eqs. [36]-39]) and the locations of the boundaries between the three regions (eqs. 40]-43]). To understand the mass-period distribution of gas giant planets in extrasolar systems found by radialvelocimetry, we need to take several additional processes into consideration. Especially, planets in the gap-limiting region would be susceptible to the type II migration, because the gas exterior to the planetary orbit blocked by the gap pushes the planet inward. Inclusion of planetary migration is our future work. Also, inclusion of core accretion processes and the gas accretion process governed by the Kelvin-Helmholtz contraction of the envelope is 
needed especially to determine the initial mass and the origin of time of our model.

Although the final masses of gas giant planets were focused on in this paper, the accretion process for reaching the final mass is also important to resolve issues relevant to planet formation. Growing giant planets dynamically affect other bodies in a planetary system. Satellites are likely to form in sub-disks around accreting gas giant planets (e.g. Canup \& Ward 2002, 2006). The long-term accretion of gas giant planets may affect the internal structure and evolution of isolated young gas giants (Marley et al. 2007). This would be important for future direct detection of young gas giants.

We are grateful to S. Ida for fruitful discussion and continuous encouragement. We also thank H. Tanaka for critical comments on our modeling. Valuable comments and suggestions from the anonymous referee were quite helpful in improving this paper. This work was supported by Ministry of Education, Culture, Sports, Science and Technology of Japan (MEXT), Grand-in-Aid for Scientific Research on Priority Areas, "Development of Extrasolar Planetary Science" (MEXT-16077202).

\section{A. Disk properties}

Typical values of the disk parameters in our model are summarized with their dependence on the semi-major axis of the planet. We assume that the disk is optically thin and in the radiative equilibrium with the stellar radiation. The disk temperature is given by (Havashi 1981)

$$
T=280 \mathrm{~K}\left(\frac{r_{\mathrm{p}}}{1 \mathrm{AU}}\right)^{-1 / 2}\left(\frac{L_{*}}{L_{\odot}}\right)^{1 / 4},
$$

where $L_{*}$ and $L_{\odot}$ is luminosities of the central star and the sun, respectively. Hence the normalized scale height (i.e., the aspect ratio of the disk) is

$$
\frac{h}{r_{\mathrm{p}}}=0.033\left(\frac{r_{\mathrm{p}}}{1 \mathrm{AU}}\right)^{1 / 4} \text {. }
$$

Note that the ratio is independent of the stellar mass because we assume the stellar luminosity is proportional to the fourth power of stellar mass, which is observationally known in the case of main sequence stars.

On the $\alpha$-prescription $(\alpha \equiv \nu /(c h))$, which is widely used to describe poorly-known turbulent viscosity in the disks (Shakura \& Sunyaev 1973), the normalized viscosity coefficient 
with the temperature distribution given by equation (A1) is

$$
\frac{\nu}{r_{\mathrm{p}}^{2} \Omega_{\mathrm{p}}}=1.1 \times 10^{-5}\left(\frac{\alpha}{10^{-2}}\right)\left(\frac{r_{\mathrm{p}}}{1 \mathrm{AU}}\right)^{1 / 2}
$$

If we assume that disk surface density is proportional to the mass of the central star, normalized surface density is

$$
\frac{\Sigma}{M_{*} / r_{\mathrm{p}}^{2}}=1.9 \times 10^{-4}\left(\frac{r_{\mathrm{p}}}{1 \mathrm{AU}}\right)^{1 / 2}\left(\frac{f}{1}\right),
$$

where $f$ is a factor relative to the surface density of the minimum mass solar nebula model when the mass of the central star equals to that of the Sun. The dissipation timescale of the disk is

$$
\frac{\tau_{\text {dep }}}{\Omega_{\mathrm{p}}^{-1}}=2 \pi \times 10^{6}\left(\frac{\tau_{\mathrm{dep}}}{10^{6} \mathrm{yr}}\right)\left(\frac{r_{\mathrm{p}}}{1 \mathrm{AU}}\right)^{-3 / 2}\left(\frac{M_{*}}{M_{\odot}}\right)^{-1 / 2},
$$

where $M_{\odot}$ is the stellar mass. The accretion rate is

$$
\frac{\dot{M}_{\mathrm{p}}}{M_{*} \Omega_{\mathrm{p}}}=4.8 \times 10^{-9}\left(\frac{\dot{M}_{\mathrm{p}}}{10^{-2} M_{\oplus} / \mathrm{yr}}\right)\left(\frac{M_{*}}{M_{\odot}}\right)^{-3 / 2}\left(\frac{r_{\mathrm{p}}}{1 \mathrm{AU}}\right)^{3 / 2}
$$

\section{B. Approximate solutions for the accretion rate in the post-gap phase}

The surface density of the gas in the accretion band at $x \sim 2 r_{\mathrm{H}}, \Sigma_{\text {acc }}$, is given by $\Sigma_{\text {vis }}$, when the accretion band exists in the region with the dynamically stable profile of surface density determined by the balance between viscous diffusion and scattering due to the planet, namely, when $2 r_{\mathrm{H}} \geq x_{\mathrm{m}}$. Substituting $x=2 r_{\mathrm{H}}$ into equation (4), one obtains

$$
\Sigma_{\mathrm{acc}}=\Sigma_{\mathrm{vis}}\left(2 r_{\mathrm{H}}\right)=\Sigma_{\infty} \exp \left(-\frac{M_{\mathrm{p}}}{M_{\text {trans }}}\right)
$$

where

$$
M_{\mathrm{trans}}=27 \pi\left(\frac{\nu}{r_{\mathrm{p}}^{2} \Omega_{\mathrm{p}}}\right) M_{*}
$$

Using equation (B1), one can rewrite $\dot{M}_{p}=\Sigma_{\text {acc }} \dot{A}$ as

$$
\dot{M}_{\mathrm{p}} \simeq \frac{M_{\text {trans }}}{t}
$$

for large $t$ and $M_{\mathrm{p}}$, if one neglects the weak dependence of $\dot{A}$ on $M_{p}$. 
When $2 r_{\mathrm{H}} \leq x_{\mathrm{m}}, \dot{M}_{p}$ depends on $M_{p}$ in a more complicated manner. As seen in equation (13), $\Sigma_{R}$ is not a simple exponential function of $M_{\mathrm{p}}$ unlike $\Sigma_{\mathrm{vis}}$. Defining a function $f\left(M_{p}\right)$ as

$$
f\left(M_{\mathrm{p}}\right) \equiv-\frac{1}{2}\left(\frac{2 r_{\mathrm{H}}}{h}-\frac{5}{4} \frac{x_{\mathrm{m}}}{h}\right)^{2}+\frac{1}{32}\left(\frac{x_{\mathrm{m}}}{h}\right)^{2}-\left(\frac{x_{\mathrm{m}}}{\ell}\right)^{-3},
$$

one can write $\Sigma_{\text {acc }}$ in the form

$$
\Sigma_{\mathrm{acc}}=\Sigma_{\mathrm{R}}\left(2 r_{\mathrm{H}}\right)=\Sigma_{\infty} \exp \left(f\left(M_{\mathrm{p}}\right)\right)
$$

Since $M_{p}$ does not change so much in the post-gap phase, one only has to integrate $\dot{M}_{p}$ in a limited range of $M_{p}$. Referring to $\Delta M_{p}$ as an increment from a mass, $M_{\mathrm{p}, 0}$ (i.e., $M_{\mathrm{p}}=$ $M_{\mathrm{p}, 0}+\Delta M_{\mathrm{p}}$ ), one expands $e^{f}$ in terms of the small quantity $\Delta M_{p}$ in such a way

$$
\begin{aligned}
\Sigma_{\mathrm{acc}}=\Sigma_{\mathrm{R}}\left(M_{\mathrm{p}, 0}+\Delta M_{\mathrm{p}}\right) & =\Sigma_{\infty} \exp \left(f\left(M_{\mathrm{p}, 0}\right)+\frac{d f}{d M_{\mathrm{p}}} \Delta M_{\mathrm{p}}\right) \\
& =\Sigma_{\mathrm{R}}\left(M_{\mathrm{p}, 0}\right) \exp \left(-\frac{\Delta M_{\mathrm{p}}}{\left(-d f / d M_{\mathrm{p}}\right)^{-1}}\right)
\end{aligned}
$$

where

$$
\frac{d f}{d M_{\mathrm{p}}}=\frac{1}{M_{\mathrm{p}}}\left(-\frac{1}{3}\left(\frac{2 r_{\mathrm{H}}}{h}\right)^{2}+\frac{11}{12^{4 / 5}}\left(\frac{2 r_{\mathrm{H}}}{h}\right)\left(\frac{\ell}{h}\right)^{3 / 5}-\frac{8}{12^{3 / 5}}\left(\frac{\ell}{h}\right)^{6 / 5}\right) .
$$

Thus one obtains

$$
\dot{M}_{\mathrm{p}} \simeq \frac{\left(-d f / d M_{\mathrm{p}}\right)^{-1}}{t}
$$

for large $t$ and $M_{\mathrm{p}}$.

\section{REFERENCES}

Alibert, Y., Mordasini, C., \& Benz, W. 2004, A\&A, 417, L25

Artymowicz, P., \& Lubow, S. H. 1996, ApJ, 467, 77

Bate, M. R., Lubow, S. H., Ogilvie, G. I., \& Miller, K. A. 2003, MNRAS, 341, 213

Bodenheimer, P., \& Pollack, J. B. 1986, Icarus, 67, 391

Boss, A. P. 2002, Earth and Planetary Science Letters, 202, 513

Boss, A. P. 1989, ApJ, 345, 554

Cameron, A. G. W. 1978, Moon and the Planets, 18, 5 
Canup, R. M., \& Ward, W. R. 2002, AJ, 124, 3404

Canup, R. M., \& Ward, W. R. 2006, Nature, 441, 834

Chandrasekhar, S. 1961, Hydrodynamic and Hydromagnetic Stability, Clarendon press

Crida, A., Morbidelli, A., \& Masset, F. 2006, Icarus, 181, 587

D’Angelo, G., Henning, T., \& Kley, W. 2002, A\&A, 385, 647

D’Angelo, G., Kley, W., \& Henning, T. 2003, ApJ, 586, 540

Guillot, T., \& Hueso, R. 2006, MNRAS, 367, 47

Hartmann, L., Calvet, N., Gullbring, E., \& D’Alessio, P. 1998, ApJ, 495, 385

Hayashi, C. 1981, Prog. Theor. Phys. Suppl., 70, 35

Ida, S., \& Lin, D. N. C. 2004, ApJ, 604, 388

Ikoma, M., \& Genda, H. 2006, ApJ, 650, 1150

Ikoma, M., Nakazawa, K., \& Emori, H. 2000, ApJ, 537, 1013

Kley, W. 1999, MNRAS, 303, 696

Lecar, M. \& Sasselov, D. D. 2003, ApJ, 99

Lin, D. N. C., \& Papaloizou, J. C. B. 1979, MNRAS, 186, 799

Lin, D. N. C., \& Papaloizou, J. C. B. 1993, Protostars and planets III, 749

Lubow, S., H., Seibert, M., \& Artymowicz, P. 1999, ApJ, 526, 1001

Lubow, S., H. \& D’Angelo, G. 2006, ApJ, 641, 526

Machida, M. N., Inutsuka, S., \& Matsumoto, T. 2006, ApJ, 649, 129

Marley, M. S., Fortney, J. J., Hubickyj, O., Bodenheimer, P., \& Lissauer, J. J. 2007, ApJ, 655,541

Masset, F., D’Angelo, G., \& Kley, W. 2006, ApJ, 652, 730

Mizuno, H. 1980, Prog. Theor. Phys., 64, 544

Pollack, J. B., Hubickyj, O., Bodenheimer, P., Lissauer, J. J., Podolak, M., \& Greenzweig, Y. 1996, Icarus, 124, 62 
Shakura, N. I., \& Sunyaev, R. A. 1973, A\&A, 24, 337

Tanigawa, T., \& Watanabe, S. 2002, ApJ, 586, 506 (TW02)

Veras, D., \& Armitage, P. J. 2004, MNRAS, 347, 613

Ward, W. R. 1997, Icarus, 126, 261

Ward, W. R. \& Harn, J. M. 2000, Protostars and planets IV, 1135

This preprint was prepared with the AAS LATEX macros v5.2. 


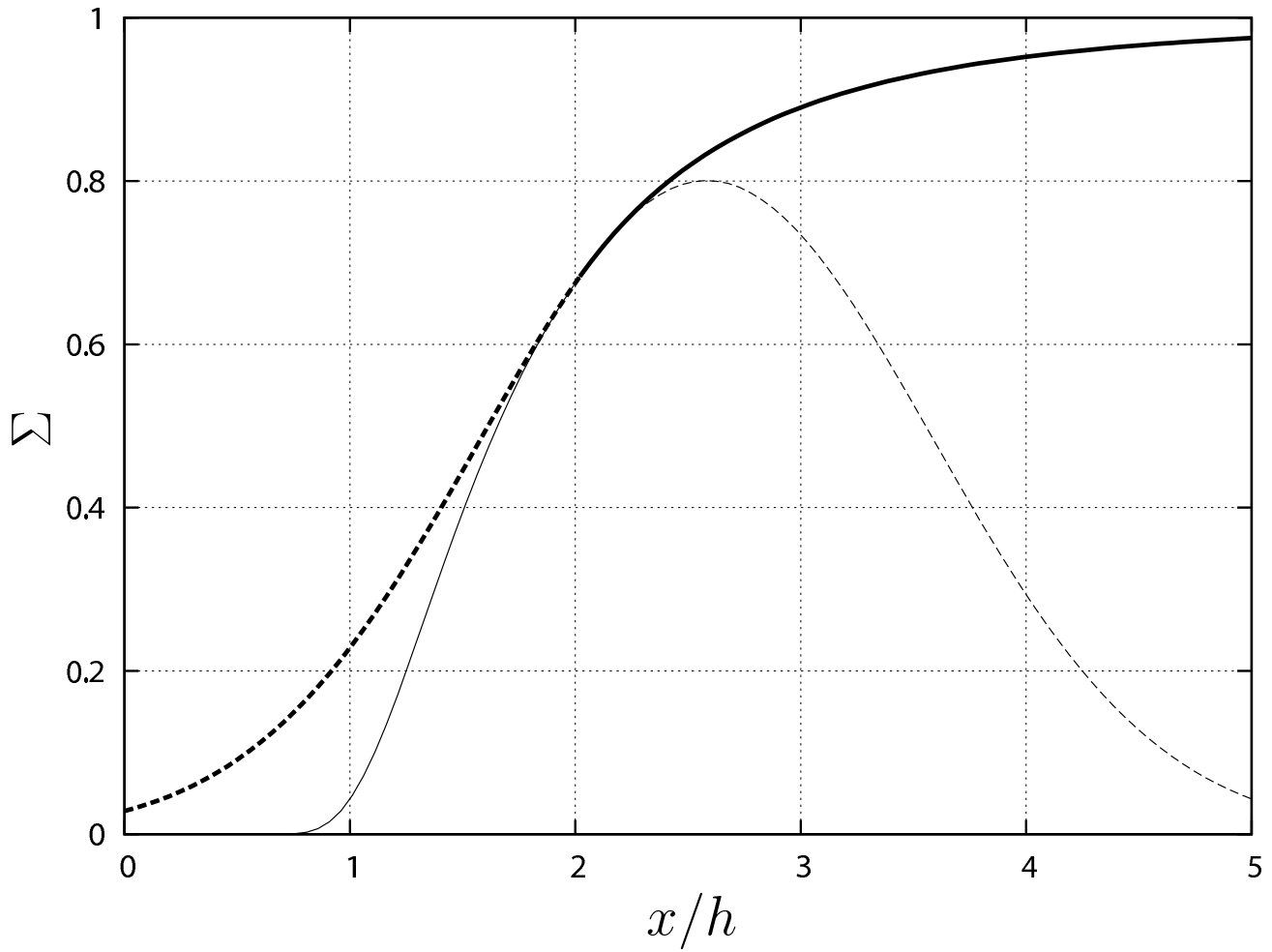

Fig. 1. - Surface density profile for $M_{\mathrm{p}} / M_{*}=10^{-3}, \nu /\left(r_{\mathrm{p}}^{2} \Omega_{\mathrm{p}}\right)=10^{-5}$, and $h / r_{\mathrm{p}}=0.1$. Solid line is $\Sigma_{\mathrm{vis}}$ given by equation (4) and dashed line is $\Sigma_{\mathrm{R}}$ given by equation (13). The two curves connect smoothly with each other at $x=x_{\mathrm{m}}$ (i.e., $x=2.08 \mathrm{~h}$ in this case). Thick line is the actual surface density we use in this paper. 

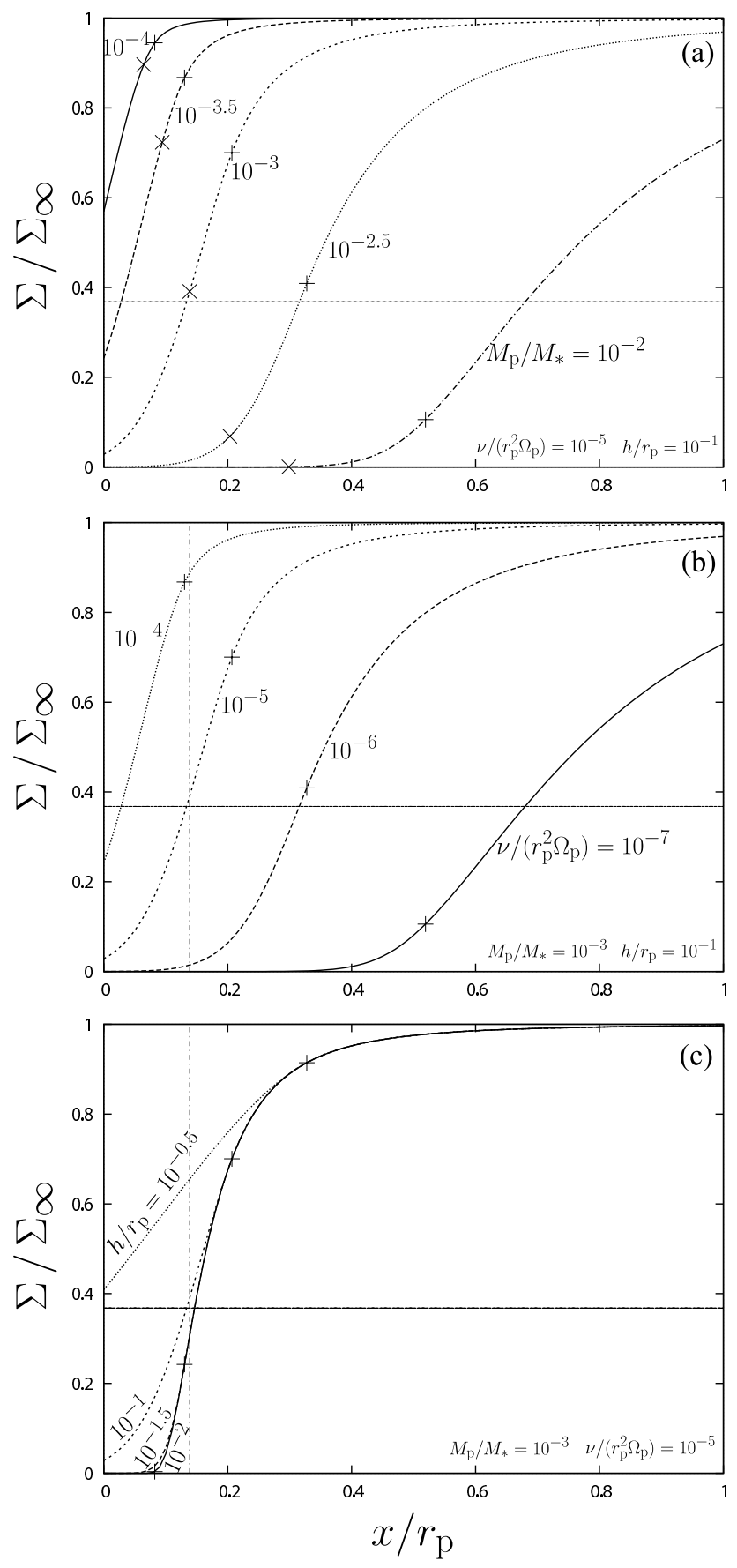

Fig. 2.- Surface density profiles as a function of distance from the planetary orbit. Top panel (a) shows planetary mass dependence $\left(M_{\mathrm{p}} / M_{*}=10^{-4}\right.$ (solid line), $10^{-3.5}, 10^{-3}, 10^{-2.5}, 10^{-2}$ (dot-dashed line)), middle panel (b) shows viscosity parameter dependence $\left(\nu /\left(r_{\mathrm{p}}^{2} \Omega_{\mathrm{p}}\right)=10^{-7}\right.$ (solid line) $10^{-6}, 10^{-5}, 10^{-4}$ (dotted line $\left.)\right)$, and bottom panel (c) shows scale height dependence $\left(h / r_{\mathrm{p}}=10^{-2}\right.$ (solid line), $10^{-1.5}, 10^{-1}, 10^{-0.5}$ (dotted line)). The standard values of the parameters are $M_{\mathrm{p}} / M_{*}=10^{-3}, \nu /\left(r_{\mathrm{p}}^{2} \Omega_{\mathrm{p}}\right)=10^{-5}$, and $h / r_{\mathrm{p}}=10^{-1}$. The position of the symbols + indicates where $x=x_{\mathrm{m}}, \times$ in the top panel and the vertical lines in the other two panels indicate where $x=2 r_{\mathrm{H}} . \Sigma_{\infty} / e$ are also shown as a horizontal line in each panel. 

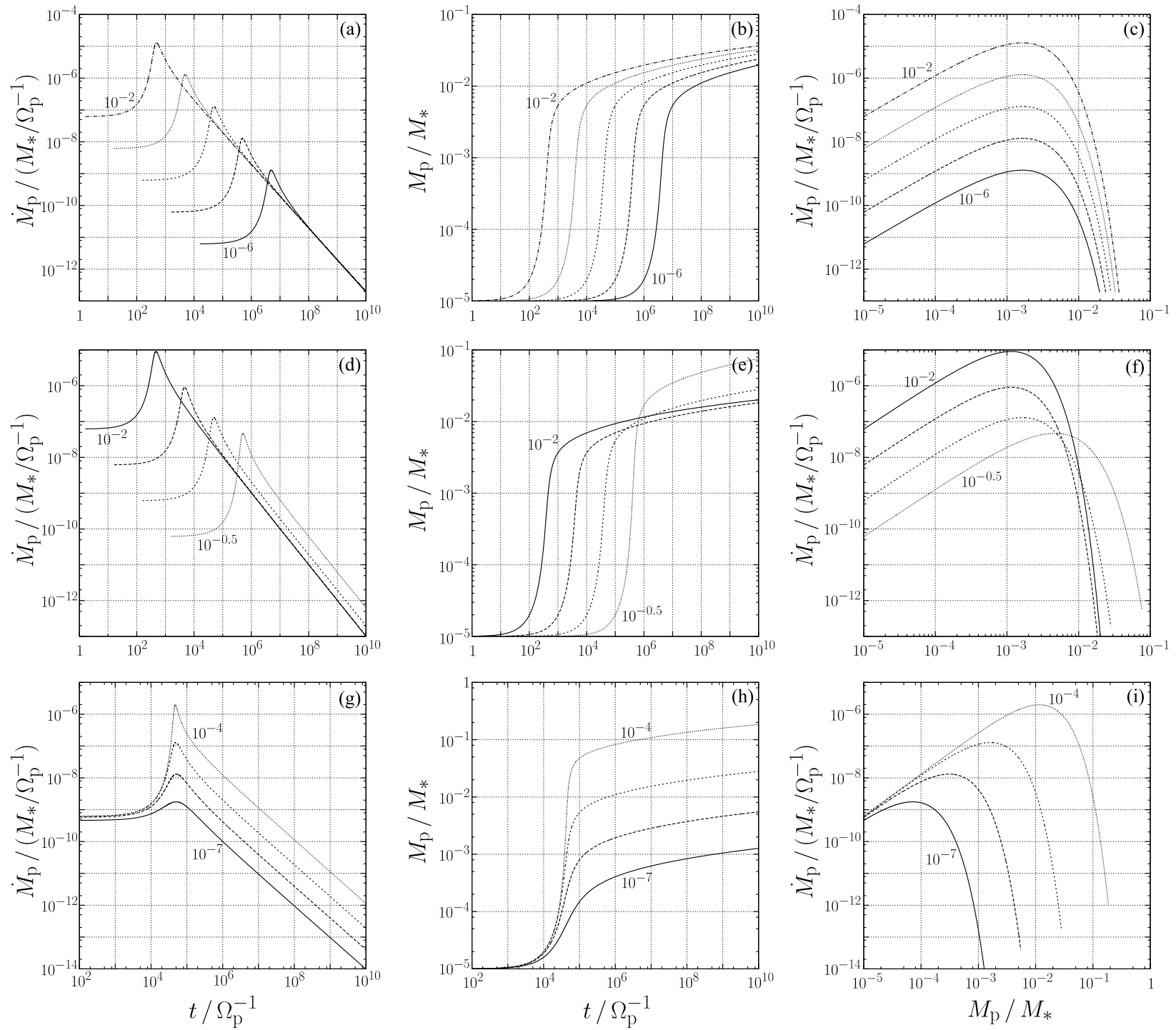

Fig. 3.- Evolution of planetary mass and accretion rate without disk dissipation. Figures in the left column show accretion rate onto the planet as a function of time, those in the central column show planetary mass as a function of time, and those in the right column show accretion rate vs planetary mass. Figures in the top row show the dependence on surface density $\left(\Sigma_{\infty} /\left(M_{*} r_{\mathrm{p}}^{-2}\right)=10^{-6}\right.$ (solid line), $10^{-5}, 10^{-4}, 10^{-3}, 10^{-2}$ (dot-dashed line)), those in the middle row show the dependence on scale height $\left(h / r_{\mathrm{p}}=10^{-2}\right.$ (solid line), $10^{-1.5}, 10^{-1}, 10^{-0.5}$ (dotted line)), and those in the bottom row show the dependence on viscosity coefficient $\left(\nu /\left(r_{\mathrm{p}}^{2} \Omega_{\mathrm{p}}\right)=10^{-7}\right.$ (solid line) $10^{-6}, 10^{-5}, 10^{-4}$ (dotted line)). The standard values of the parameters are $\Sigma_{\infty} /\left(M_{*} r_{\mathrm{p}}^{-2}\right)=10^{-4}, \nu /\left(r_{\mathrm{p}}^{2} \Omega_{\mathrm{p}}\right)=10^{-5}$, and $h / r_{\mathrm{p}}=10^{-1}$. 

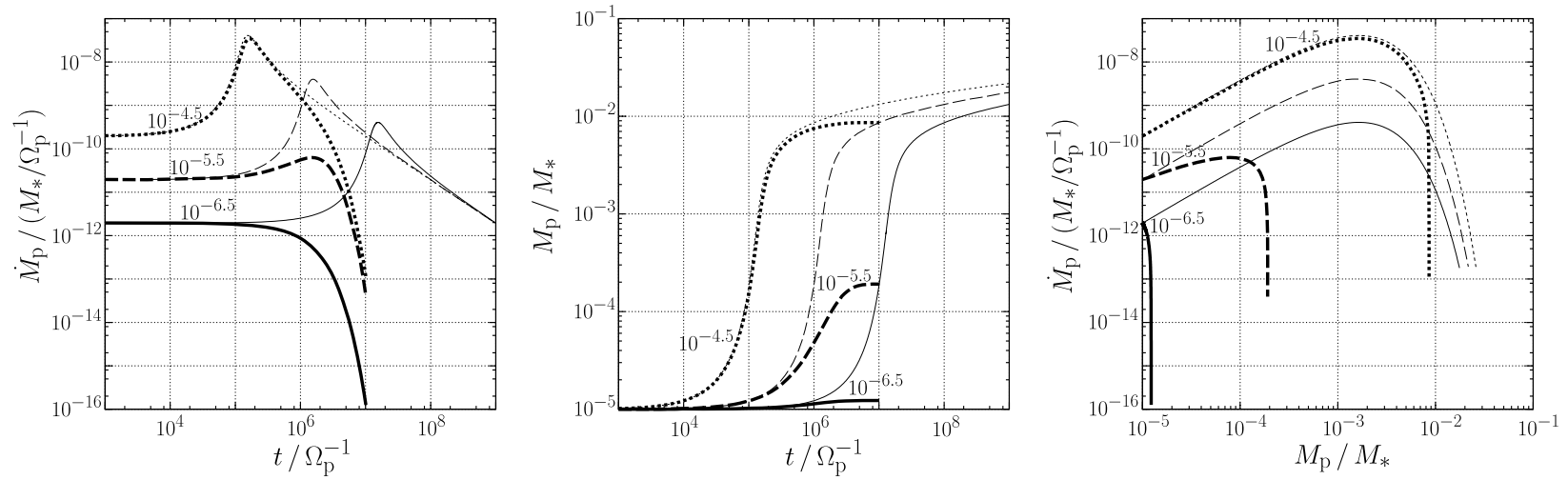

Fig. 4. - Evolution of planetary mass and accretion rate with disk dissipation when $h / r_{\mathrm{p}}=$ $0.1, \nu /\left(r_{\mathrm{p}}^{2} \Omega_{\mathrm{p}}\right)=10^{-5}$. Three panels are the same axis with Fig. 3. Three thick lines show different initial surface densities $\left(\Sigma_{\text {init }} /\left(M_{*} r_{\mathrm{p}}^{-2}\right)=10^{-6.5}\right.$ (solid line), $10^{-5.5}$ (long-dashed line), $10^{-4.5}$ (short-dashed line)) and evolutions without disk dissipation are also shown as thin lines.

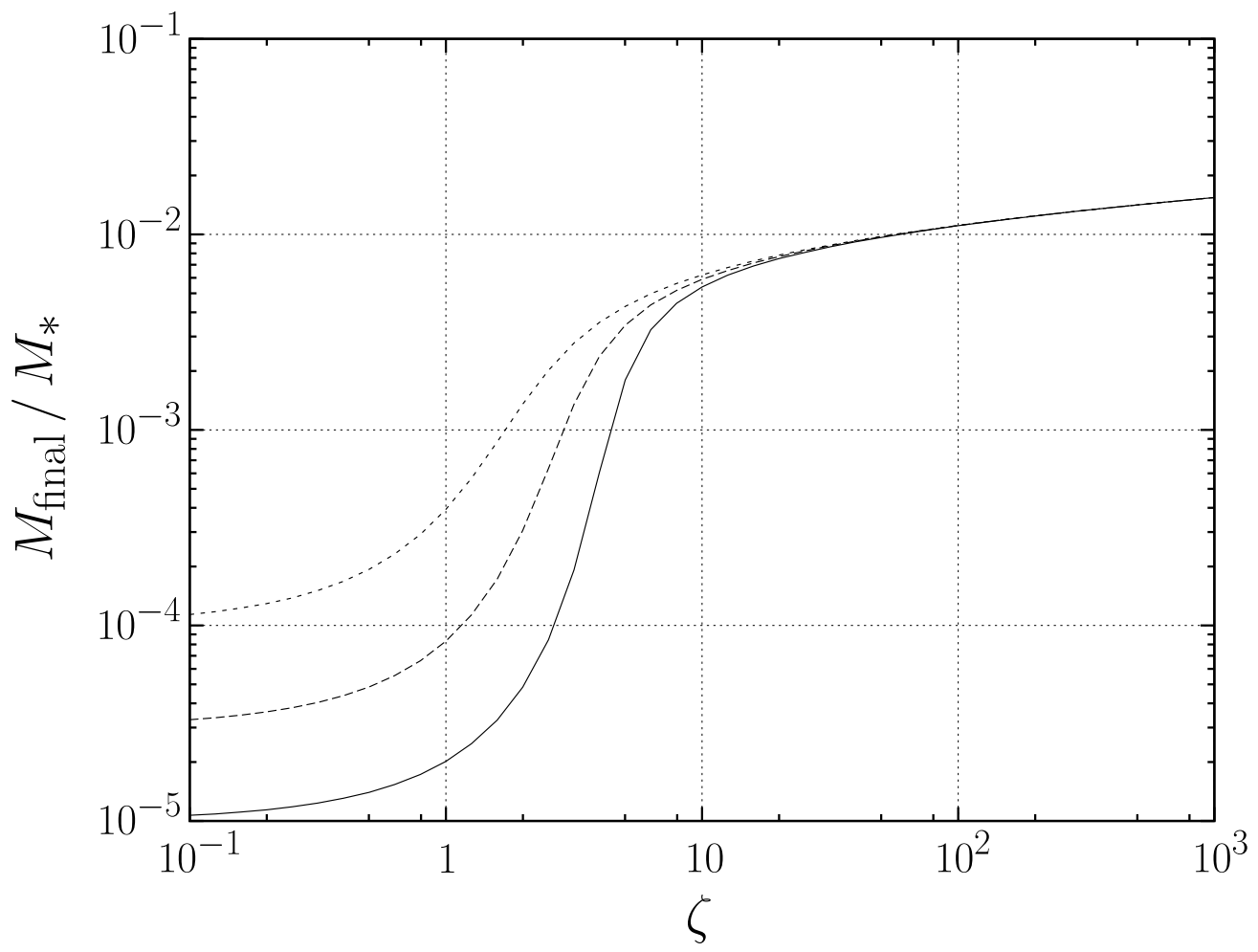

Fig. 5.- Final mass of planets as a function of $\zeta$ (defined at eq. 24]) in the case with $h / r_{\mathrm{p}}=$ $0.1, \nu /\left(r_{\mathrm{p}}^{2} \Omega_{\mathrm{p}}\right)=10^{-5}$. The three lines are different initial planetary mass $\left(M_{\mathrm{p}, \text { init }} / M_{*}=\right.$ $\left.1 \times 10^{-5}, 3 \times 10^{-5}, 1 \times 10^{-4}\right)$. 

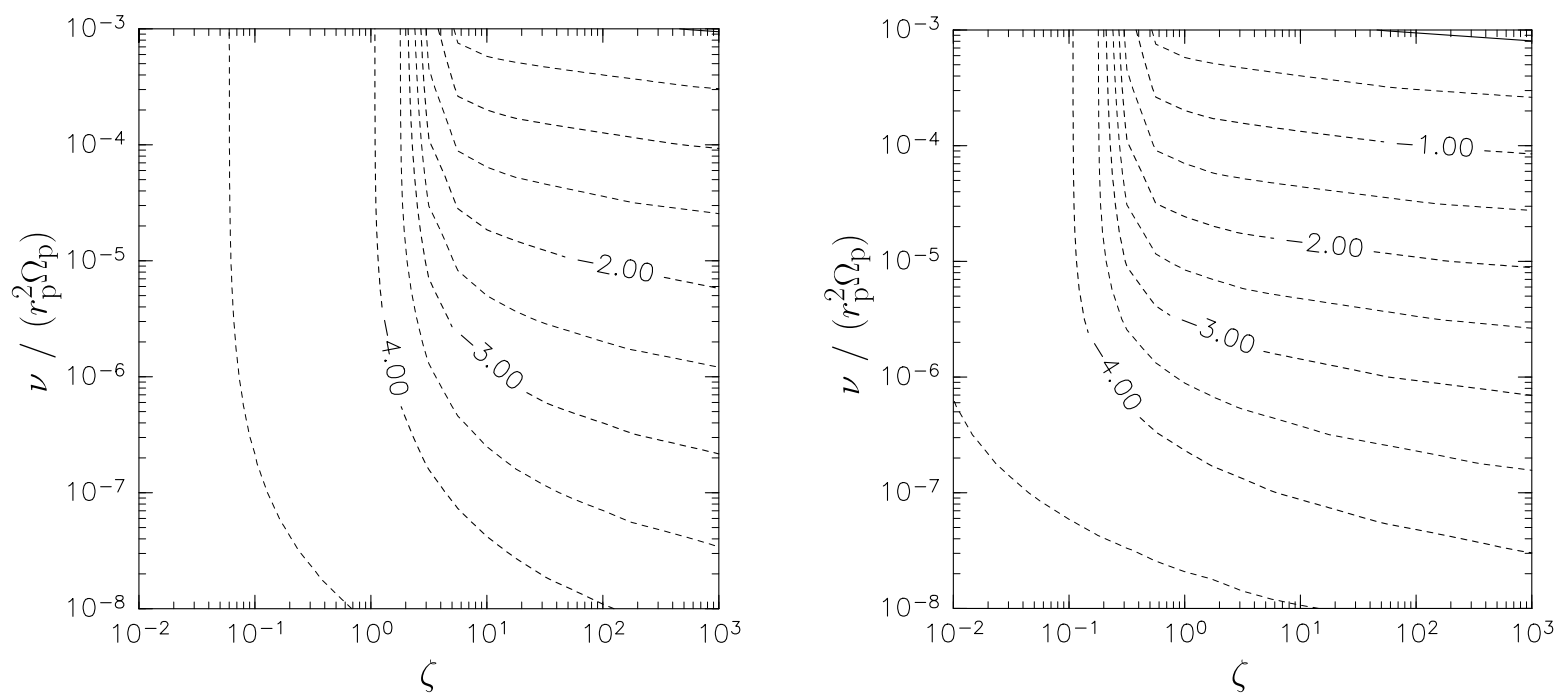

Fig. 6.- Contour lines of $\log \left(M_{\text {final }} / M_{*}\right)$ as a function of $\zeta$ and $\nu /\left(r_{\mathrm{p}}^{2} \Omega_{\mathrm{p}}\right)$. Left panel is when $h=0.1 r_{\mathrm{p}}$, right panel is when $h=10^{-1.5} r_{\mathrm{p}}$. The initial mass is set to $10^{-5} M_{*}$.
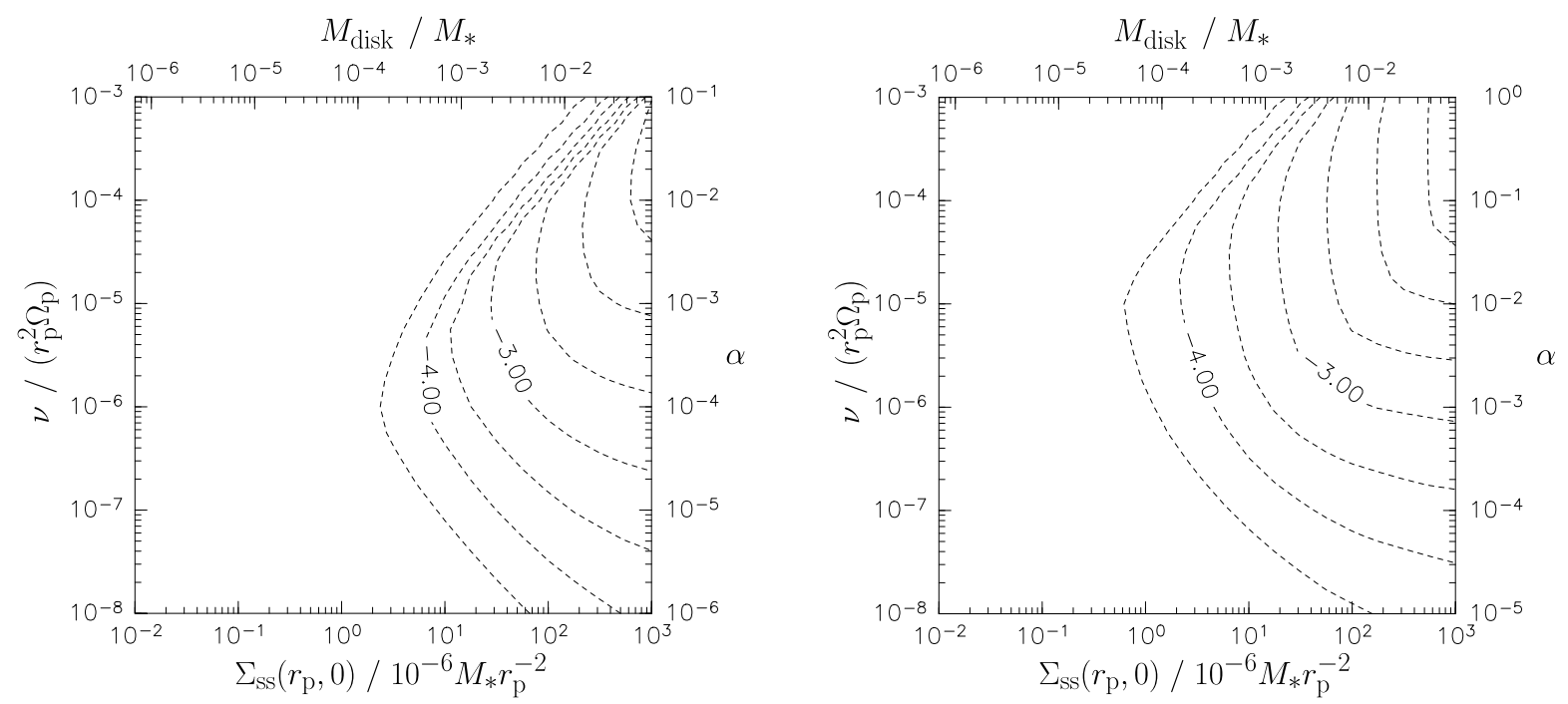

Fig. 7.- Contour lines of $\log \left(M_{\text {final }} / M_{*}\right)$ when global viscous evolution is considered in the case with $\tau_{\text {dep }}=10^{6}$. Horizontal axis is normalized surface density times $10^{6}$ (i.e., it corresponds to $\zeta$ when $\left.\Sigma_{\infty \text {,init }}=\Sigma_{\mathrm{ss}}\left(r_{\mathrm{p}}, 0\right)\right)$ and corresponding disk mass assuming $R_{\text {out }}=$ $10 r_{\mathrm{p}}$ is also shown on the top. Vertical axis is normalized viscosity and corresponding $\alpha$ is also shown on the right. Left panel shows the case with $h / r=0.1$ and right panel shows when $h / r=0.032$. 


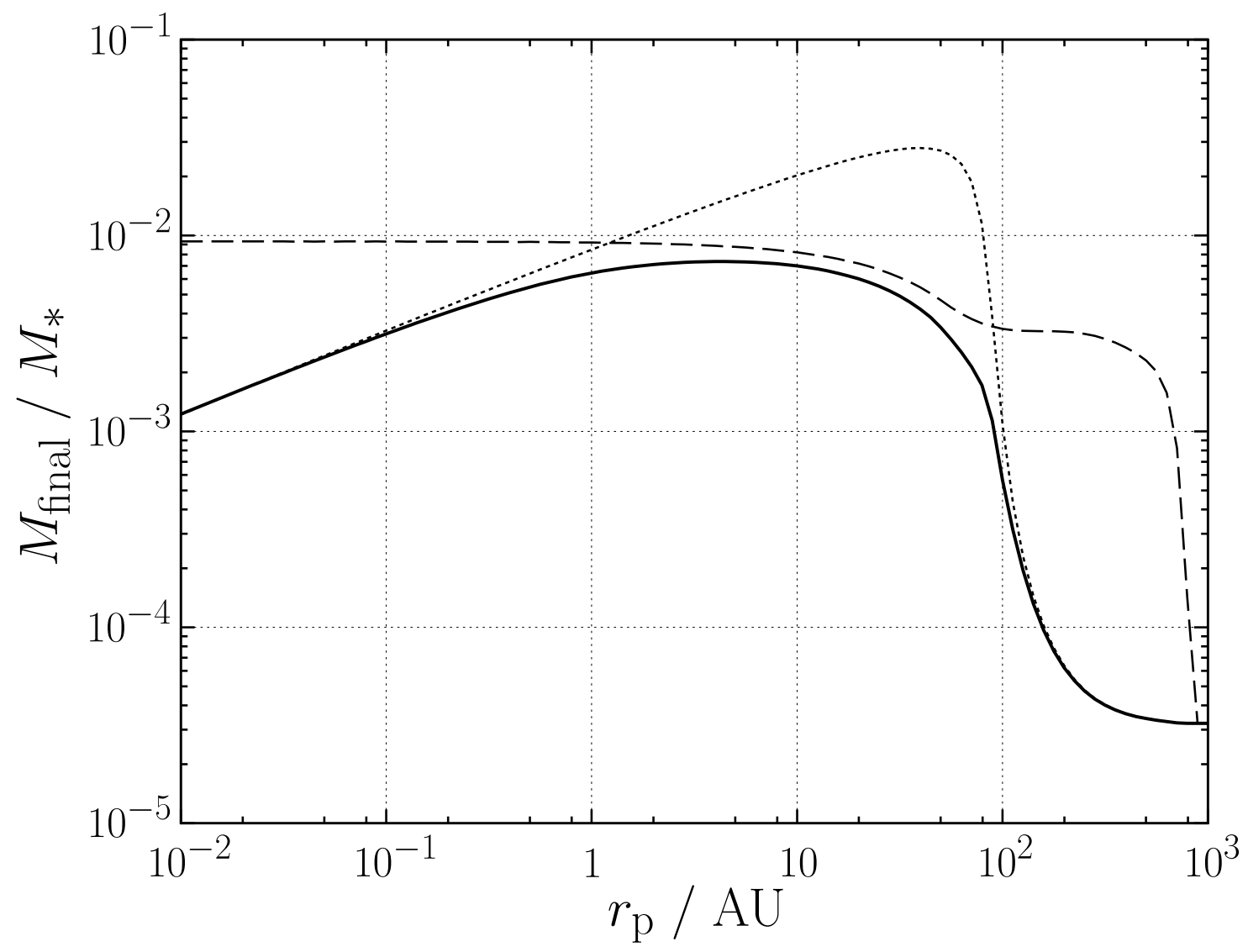

Fig. 8. - Final mass of planets as a function of semi-major axis in AU. Solid line shows standard case: $\alpha=0.01, h / r=0.032$ at $1 \mathrm{AU}, \tau_{\text {dep }}=10^{6} \mathrm{yr}, M_{\text {disk }}=1.3 \times 10^{-2} M_{*}, R_{\text {out }}=$ 100AU. Dashed line shows $M_{\text {p,disk }}$ (i.e., mass when all viscous-accreting gas is assumed to accrete onto planets), and dotted line shows $M_{\mathrm{p}, \mathrm{ss}}$ (i.e., mass when the global viscous evolution with self-similar solution is not assumed). Initial mass of the planets is set as $3.2 \times 10^{-5} M_{*}$, which corresponds to 10 Earth masses in the solar mass system. 


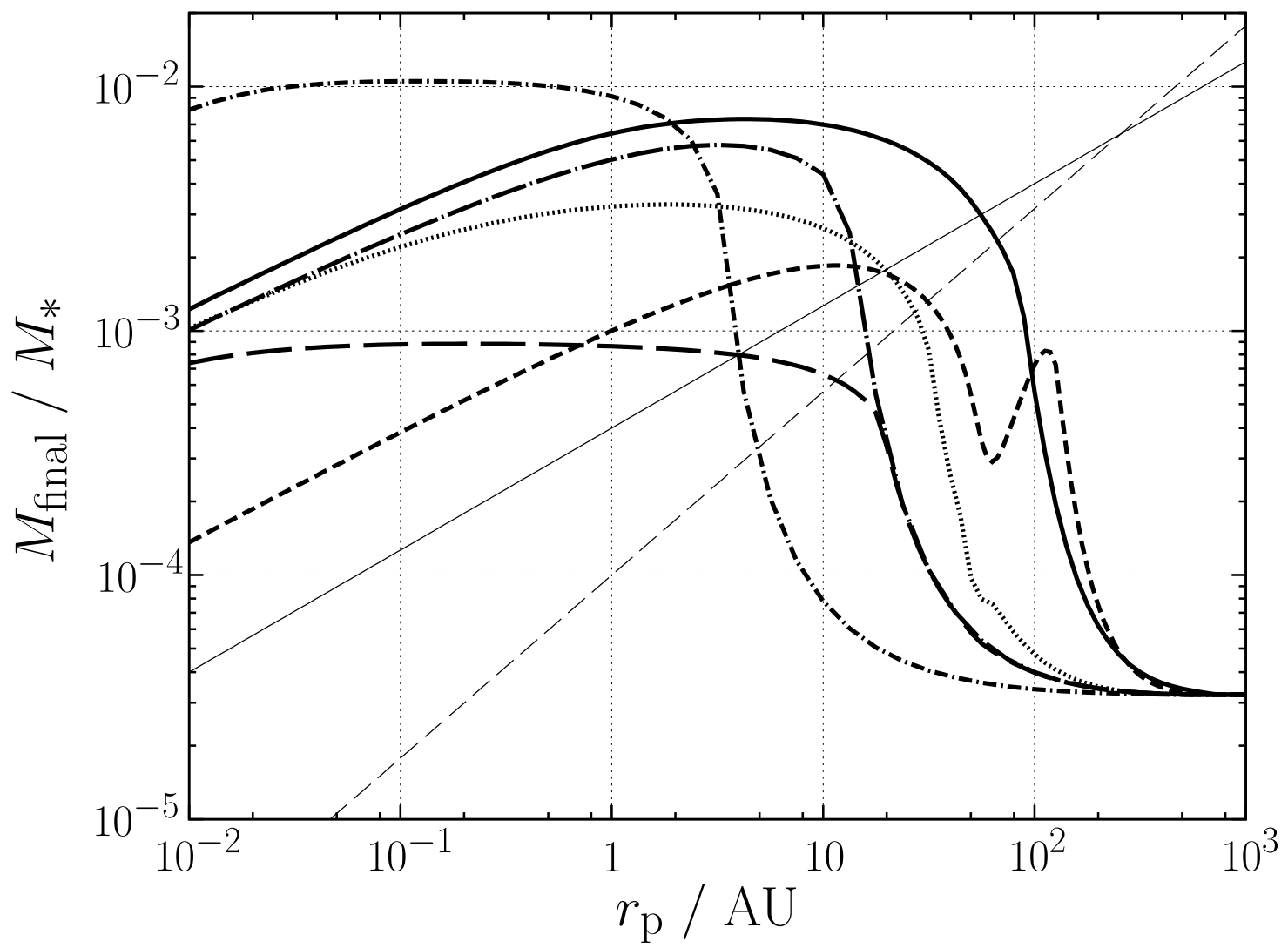

Fig. 9.- Final mass of planets as a function of semi-major axis in AU for several disk models. Thick solid line shows standard case as described in Figure 8. Thick long-dashed line shows $M_{\text {disk }}$ is 10 times smaller case, thick short-dashed line shows viscous $\alpha$ is 10 times smaller than the standard case, thick dotted line shows $\tau_{\text {dep }}$ is 10 times shorter case, thick dot-short-dashed line shows the case when scale height is 3 times higher, and thick dot-longdashed line shows the case when gas accretion rate is reduced by a factor of 10 . Thin solid line and thin dashed line show the masses determined by the viscous and thermals condition $(\alpha=0.01)$, respectively. 
Table 1. List of notations

\begin{tabular}{|c|c|c|}
\hline Variables & Meaning & Definition \\
\hline$M_{\mathrm{p}}$ & Planetary mass & \\
\hline$M_{*}$ & Mass of the central star & \\
\hline$M_{\text {disk }}$ & Disk mass at the initial condition & $\int_{0}^{\infty} 2 \pi r \Sigma_{\mathrm{SS}}(r, 0) d r$ \\
\hline$M_{\text {final }}$ & Final mass of a planet & Eq. 23 \\
\hline$M_{\text {final,gap }}$ & Final mass of a planet in the gap-limiting region & $\mathrm{Eq} \cdot 36$ \\
\hline$M_{\text {final,diff }}$ & Final mass of a planet in the diffusion-limiting region & Eqs. 38 and 39 \\
\hline$M_{\text {trans }}$ & Planetary mass when $\Sigma_{\text {acc }}$ is $(1 / e) \Sigma_{\infty}$ in the case of $2 r_{\mathrm{H}} \geq x_{\mathrm{m}}$ & $\mathrm{Eq} \cdot \mathrm{B} 2$ \\
\hline$M_{\mathrm{p}, \text { init }}$ & Initial planetary mass & \\
\hline$M_{\mathrm{p}, \mathrm{crit}}$ & Maximum planetary mass that does not violate a geometrical limit for eq. 17] & Eq. 48 \\
\hline$M_{\mathrm{p}, \text { local }}$ & Final mass if $\dot{M}_{\mathrm{p}}$ is assumed to be $\dot{M}_{\mathrm{p}, \text { local }}$ & Eq. 35 \\
\hline$M_{\mathrm{p}, \mathrm{disk}}$ & Final mass if $\dot{M}_{\mathrm{p}}$ is assumed to be $\dot{M}_{\text {disk }}$ & Eq. 35 \\
\hline$M_{\mathrm{p}, \mathrm{vis}}$ & Planetary mass when viscous condition for gap opening is fulfilled & Eq. 44 \\
\hline$\dot{M}_{\mathrm{p}}$ & Accretion rate onto a planet & Eqs. 16 or 32 \\
\hline$\dot{M}_{\mathrm{p}, \text { local }}$ & Accretion rate onto a planet using eqs. [14, [16, and 26 & see 4.3 .1 \\
\hline$\dot{M}_{\text {disk }}$ & Radial mass flux of the disk due to viscous evolution & Eq. 30 \\
\hline$\Sigma_{\infty}$ & Unperturbed surface density at planet's orbit & Eqs. 4, 13, 21, 26] \\
\hline$\Sigma_{\text {acc }}$ & Surface density at the accretion band $\left(x=2 r_{\mathrm{H}}\right)$ & Eq. 16] \\
\hline$\Sigma_{\text {vis }}$ & Surface density determined by the balance between viscous and gravitational torques & Eq. \\
\hline$\Sigma_{\mathrm{R}}$ & Surface density determined by marginally stable state of the Rayleigh condition & Eq. 13 \\
\hline$\Sigma_{\mathrm{ss}}$ & Surface density of self-similar solution for viscous evolving disk & Eq. 27 \\
\hline$\Sigma_{\infty, \text { init }}$ & Initial unperturbed surface density at planet's orbit & Eq. 21 \\
\hline$r_{\mathrm{p}}$ & Semi-major axis of a planet & \\
\hline$r_{\mathrm{b}}$ & Boundary position between the gap-limiting region and the diffusion-limiting region & see 4.3 .3 \\
\hline$r_{\mathrm{e}}$ & Boundary position between the diffusion-limiting region and the no-growth region & see 4.3 .3 \\
\hline$\ell$ & Position where $\Sigma_{\text {vis }}$ is $(1 / e) \Sigma_{\infty}$ & Eqs. 4 or 5 \\
\hline$x_{\mathrm{m}}$ & Position where the Rayleigh condition is marginally fulfilled for $\Sigma_{\text {vis }}$ & Eq. 10 \\
\hline$\tau_{\text {div }}$ & Time when the accretion rate is the maximum & Eq. 19 \\
\hline$\tau_{\text {dep }}$ & Exponential depletion timescale of a disk & Eq. 21 \\
\hline$\tau_{\text {vis }}$ & Viscous evolution timescale of a disk & Eq. 29] \\
\hline$\tau_{\text {lifetime }}$ & Effective disk lifetime (i.e., shorter one of $\tau_{\text {dep }}$ and $\tau_{\text {vis }}$ ) & Eq. 34 \\
\hline$\zeta$ & Normalized parameter (Surface density times disk depletion time) & Eq. 24 \\
\hline
\end{tabular}

